\title{
The Integrin Signaling Network Promotes Axon Regeneration via the Src-Ephexin-RhoA GTPase Signaling Axis
}

\author{
Yoshiki Sakai, Mayuka Tsunekawa, Kohei Ohta, Tatsuhiro Shimizu, ${ }^{\circledR}$ Strahil Iv. Pastuhov, Hiroshi Hanafusa, \\ Naoki Hisamoto, and Kunihiro Matsumoto \\ Division of Biological Science, Graduate School of Science, Nagoya University, Nagoya 464-8602, Japan
}

\begin{abstract}
Axon regeneration is an evolutionarily conserved process essential for restoring the function of damaged neurons. In Caenorhabditis elegans hermaphrodites, initiation of axon regeneration is regulated by the RhoA GTPase-ROCK (Rho-associated coiled-coil kinase)-regulatory nonmuscle myosin light-chain phosphorylation signaling pathway. However, the upstream mechanism that activates the RhoA pathway remains unknown. Here, we show that axon injury activates TLN-1/talin via the cAMP-Epac (exchange protein directly activated by cAMP)-Rap GTPase cascade and that TLN-1 induces multiple downstream events, one of which is integrin inside-out activation, leading to the activation of the RhoA-ROCK signaling pathway. We found that the nonreceptor tyrosine kinase Src, a key mediator of integrin signaling, activates the Rho guanine nucleotide exchange factor EPHX-1/ephexin by phosphorylating the Tyr-568 residue in the autoinhibitory domain. Our results suggest that the C. elegans integrin signaling network regulates axon regeneration via the Src-RhoGEF-RhoA axis.
\end{abstract}

Key words: axon regeneration; C. elegans; inside-out; integrin; RhoA; Src

\section{Significance Statement}

The ability of axons to regenerate after injury is governed by cell-intrinsic regeneration pathways. We have previously demonstrated that the Caenorhabditis elegans RhoA GTPase-ROCK (Rho-associated coiled-coil kinase) pathway promotes axon regeneration by inducing MLC-4 phosphorylation. In this study, we found that axon injury activates TLN-1/talin through the cAMP-Epac (exchange protein directly activated by cAMP)-Rap GTPase cascade, leading to integrin inside-out activation, which promotes axonal regeneration by activating the RhoA signaling pathway. In this pathway, SRC-1/Src acts downstream of integrin activation and subsequently activates EPHX-1/ephexin RhoGEF by phosphorylating the Tyr-568 residue in the autoinhibitory domain. Our results suggest that the $C$. elegans integrin signaling network regulates axon regeneration via the Src-RhoGEF-RhoA axis.

Received Sep. 19, 2020; revised Apr. 5, 2021; accepted Apr. 7, 2021.

Author contributions: Y.S., N.H., and K.M. designed research; Y.S., M.T., K.O., T.S., S.I.P., and H.H. performed research; Y.S., N.H., and K.M. analyzed data; Y.S. and K.M. wrote the paper.

This work was supported by grants from the Ministry of Education, Culture and Science of Japan (to S.I.P., H.H., and K.M.) and the Project for Elucidating and Controlling Mechanisms of Aging and Longevity from the Japan Agency for Medical Research and Development under Grant JP20gm5010001 (to N.H.). Some strains were provided by the Caenorhabditis Genetic Center (CGC), which is funded by National Institutes of Health Office of Research Infrastructure Programs (Grant P40-0D-10440). We thank the (GC, National Bio-Resource Project and C. elegans Knockout Consortium for materials.

The authors declare no competing financial interests.

Correspondence should be addressed to Kunihiro Matsumoto at g44177a@nucc.cc.nagoya-u.ac.jp or Naoki Hisamoto at i45556a@nucc.nagoya-u.ac.jp.

https://doi.org/10.1523/JNEUROSCI.2456-20.2021

Copyright $\odot 2021$ Sakai et al.

This is an open-access article distributed under the terms of the Creative Commons Attribution 4.0 International license, which permits unrestricted use, distribution and reproduction in any medium provided that the original work is properly attributed.

\section{Introduction}

The ability of neurons to regenerate damaged axons is essential for functional recovery. The regeneration of axons after injury requires the induction of multiple intracellular changes. To achieve this regeneration, the axon undergoes the following processes: local cytoskeletal reorganization to promote growth cone formation, lipid and protein transport for axon outgrowth, and activation of transcription factors that trigger regenerative programs (He and Jin, 2016). Therefore, manipulation of these processes could be an attractive strategy for therapeutic intervention to improve neuronal regeneration. However, the underlying molecular mechanisms that regulate these processes are not fully understood.

Following nerve injury, the end of the damaged axon is transformed into a growth cone-like structure. This is a crucial step in mounting a successful regenerative response. In particular, the cytoskeletal reorganization that accompanies the generation of the new growth cone is essential for the intrinsic ability to 
A

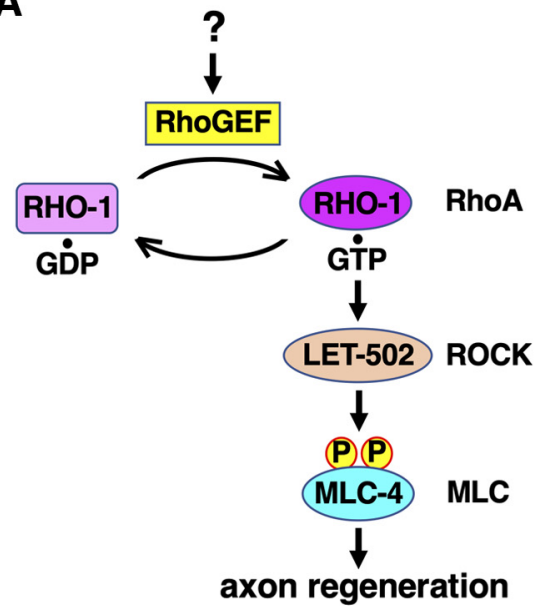

B

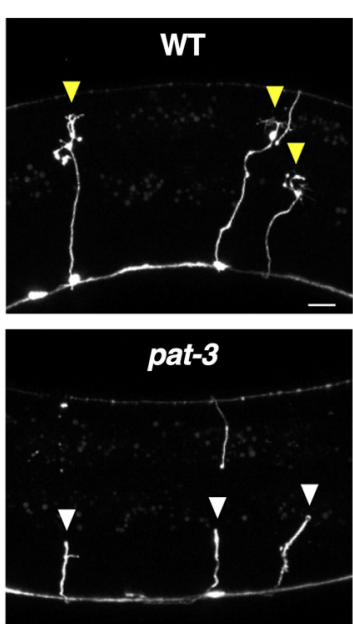

C

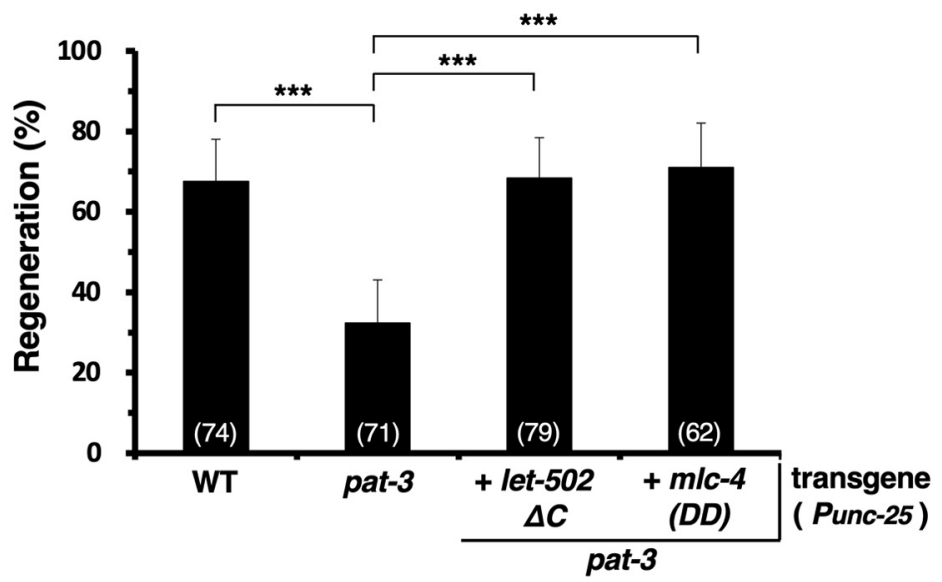

Figure 1. PAT-3 functions in the RH0-1-MLC-4 phosphorylation pathway to regulate axon regeneration. $\boldsymbol{A}$, The RH0-1/RhoA-LET-502/ROCK-MLC phosphorylation signaling pathway regulating axon regeneration in C. elegans. $\boldsymbol{B}$, Representative D-type motor neurons in wild-type (WT) and pat-3 mutant animals $24 \mathrm{~h}$ after laser surgery. In wildtype animals, severed axons exhibited regenerating growth cones (yellow arrowheads). In pat-3 mutants, the proximal ends of axons failed to regenerate (white arrowheads). Scale bar, $10 \mu \mathrm{m}$. C, Percentages of axons that initiated regeneration $24 \mathrm{~h}$ after laser surgery in the young adult stage. The numbers of axons examined are shown. Error bars indicate $95 \%$ confidence intervals. $* * * p<0.001$, as determined by Fisher's exact test.

regenerate (Bradke et al., 2012). Growth cone formation and axonal regeneration require a number of changes in actin cytoskeletal dynamics and alterations in microtubule stability (Coles and Bradke, 2015). During axon outgrowth, the Rho family of guanosine triphosphatases (GTPases), such as RhoA, Racl, and Cdc42, plays an important role in transducing signals that lead to the reorganization of the actin cytoskeleton within the growth cone (Hall, 1998). Rho GTPases are molecular switches that cycle between an inactive GDP-bound form and a GTP-bound active conformation (Boguski and McCormick, 1993). The formation of active Rho GTPases is accelerated by guanine nucleotide exchange factors (GEFs; Cerione and Zheng, 1996). Activated Rho GTPases can interact with effector proteins, which, in turn, trigger a variety of cellular responses. Among RhoA effectors, Rho-associated coiled-coil kinase (ROCK) is known to play a key role in actin organization through myosin activation (Bishop and Hall, 2000).

The nematode Caenorhabditis elegans is a valuable model for elucidating the molecular mechanisms involved in axon regeneration (Yanik et al., 2004). Genetic studies in C. elegans have identified common biological pathways that use conserved molecules to regulate regeneration (Hammarlund et al., 2009; Nix et al., 2011; Bejjani and Hammarlund, 2012). We have recently reported that the $C$. elegans RhoA homolog RHO-1 promotes axon regeneration of motor neurons by activating the downstream effector LET-502, the C. elegans homolog of ROCK (Shimizu et al., 2018). Activated LET-502 phosphorylates nonmuscle myosin light-chain (MLC) MLC-4. Phosphorylation of MLC activates the $\mathrm{Mg}^{2+}$-dependent ATPase activity of nonmuscle myosin II, resulting in actin-myosin interaction (Amano et al., 1996). Thus, the RHO-1/RhoA-LET-502/ROCK pathway positively regulates $C$. elegans axon regeneration through MLC-4 phosphorylation (Fig. 1A). However, it is still unknown how the RHO-1 pathway is activated on axon injury.

Integrin signaling is one well characterized pathway that regulates the activation status of Rho GTPases (Huveneers and Danen, 2009). Integrins are $\alpha-\beta$-heterodimeric transmembrane receptors that mediate cell-extracellular matrix (ECM) interactions (Hynes, 1987). Upon binding to ECM proteins, integrins can transduce signals that activate Rho GTPases, thereby initiating cytoskeletal rearrangement. Integrin function is regulated by inside-out and outside-in signaling (Kim et al., 2011). Inside-out signaling converts the extracellular domain into a high-affinity receptor, allowing interaction with a ligand, which alters the activity of intracellular components such as protein kinases and GTPases (outside-in signaling). Thus, integrins are bidirectional signaling receptors that transmit information into and out of cells. A critical step in the activation of inside-out signaling is the binding of the cytoskeletal protein talin to the cytoplasmic domain of $\beta$-integrin. This interaction leads to the dissociation of the integrin $\alpha$-and $\beta$-transmembrane domains that modulate downstream signaling (Kim et al., 2003).

In the mammalian nervous system, integrins are involved in axon growth, synaptogenesis, and axon regeneration (Nikonenko et al., 2003; Eva and Fawcett, 2014). The C. elegans integrins INA-1/ integrin $\alpha$ and PAT-3/integrin $\beta$ also participate in axon regeneration (Pastuhov et al., 2016; Hisamoto et al., 2019). In this study, we investigated the relationship between integrin signaling and RhoA activation in the regulation of axon regeneration in C. elegans. We found that TLN-1/talin-mediated integrin inside-out activation promotes axon regeneration by activating the $\mathrm{RHO}-1 / \mathrm{RhoA}$ signaling pathway. We showed that nonreceptor tyrosine kinase Src, an important effector downstream of integrin, phosphorylates EPHX-1/ ephexin RhoGEF at the Tyr-568 residue located in the autoinhibitory region. This phosphorylation relieves the autoinhibitory regulation of EPHX-1, leading to its activation. These results suggest that the integrin-Src-RhoGEF cascade activates the RhoA-ROCK pathway in axon regeneration.

\section{Materials and Methods}

C. elegans strains. The C. elegans strains used in this study are listed in Table 1. All strains were maintained on nematode growth medium 
Table 1. Strains used in this study

\begin{tabular}{|c|c|}
\hline Strain & Genotype \\
\hline KU501 & juls76 II \\
\hline KU1265 & juls76 II; pat-3(gk804163) III \\
\hline KU1356 & juls76 II; pat-3(gk804163) III; kmEx1405 [Punc-25::let-502 LC] \\
\hline KU1357 & juls76 II; pat-3(gk804163) III; kmEx1406 [Punc-25::venus::m/c-4(DD)] \\
\hline KU1358 & tIn-1(e259) I; juls76 II \\
\hline KU1359 & tIn-1(e259) l; juls76 II; pat-3(gk804163) III \\
\hline KU1360 & t/n-1(e259) l; juls76 II; kmEx1405 [Punc-25::let-502 $\Delta C]$ \\
\hline KU1361 & $\operatorname{tln}-1($ e259) I; juls76 Il; kmEx1406 [Punc-25::venus:::mlc-4(DD)] \\
\hline KU1362 & juls76 II; pat-3(D768R) III \\
\hline KU1363 & tIn-1(e259) I; juls76 II; pat-3(D768R) III \\
\hline KU1364 & juls76 II; ina-1(R1114D) III \\
\hline KU1365 & tIn-1(e259) I; juls76 II; ina-1(R1114D) III \\
\hline KU1366 & juls76 II; pat-3(gk804163; D768R) III \\
\hline KU1367 & juls76 II; pat-3(gk804163) ina-1(R1114D) III \\
\hline KU1368 & juls76 II; rap-1(pk2082) IV \\
\hline KU1369 & juls76 II; rap-2(gk11) V \\
\hline KU1370 & tln-1(e259) I; juls76 II; rap-2(gk11) V \\
\hline KU1371 & $\operatorname{tln}-1(L 347 A)$ I; juls76 II; rap-2(gk11) V \\
\hline KU1372 & $\operatorname{tln}-1(L 347 A:: C A A X)$ I; juls76 II; rap-2(gk11) V \\
\hline KU1373 & juls76 II; pat-3(D768R) III; rap-2(gk11) V \\
\hline KU1374 & 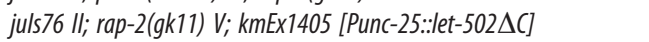 \\
\hline KU1375 & juls76 II; deb-1(gk329549) IV \\
\hline KU1376 & 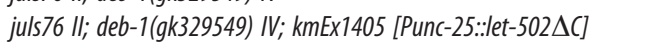 \\
\hline KU1377 & juls76 II; deb-1(gk329549) IV; kmEx1406 [Punc-25::venus::m/c-4(DD)] \\
\hline KU1378 & juls76 II; epac-1(tm3203) III \\
\hline KU1379 & juls76 II; epac-1(tm3203) III; rap-2(gk11) V \\
\hline KU1380 & juls76 II; epac-1(tm3203) III; kmEx1380 [Punc-25::rap-2(G12V)] \\
\hline KU1381 & juls76 II; epac-1(tm3203) III; kmEx1381 [Punc-25::rap-2(S17A)] \\
\hline KU1382 & juls76 II; epac-1(G84E; G440D) III line \#1 \\
\hline KU1383 & juls76 II; ерас-1(G84E; G440D) III line \#2 \\
\hline KU1384 & ephx-1(Y568F) juls76 II \\
\hline KU1385 & ephx-1(Y568E) juls76 II \\
\hline KU1386 & 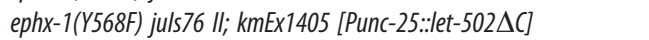 \\
\hline KU1387 & juls76 Il; kmEx1387 [Punc-25::ephx-1 $1 \Delta N]$ \\
\hline KU722 & src-1(cj293)/hT2[bli-4(e937) let-?(q782) qls48] (I;ill); juls76 II \\
\hline KU1388 & 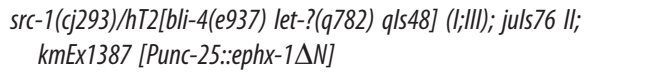 \\
\hline
\end{tabular}

plates and fed with bacteria of the OP50 strain by the standard method, as described previously (Brenner, 1974).

Plasmids. Punc-25::let-502 $\Delta C$ and Punc-25::venus::mlc-4(DD) plasmids were described previously (Shimizu et al., 2018). Punc-25::rap-2 (G12V) and Punc-25::rap-2(S17A) plasmids were generated by inserting the rap-2 cDNA isolated from a cDNA library into the pSC325 vector, followed by oligonucleotide-directed inverse PCR. The FLAG-RAP-2 (G12V or S17A) plasmid was generated by inserting rap-2(G12V) or rap-2(S17A) cDNA into the pCMV-FLAG vector. The Punc-25::ephx$1 \Delta N$ plasmid was generated by inserting the $e p h x-1 \mathrm{cDNA}$ (isoform a) isolated from a cDNA library into the pSC325 vector, followed by oligonucleotide-directed inverse PCR. GFP-EPHX-1(557-656) and GFPTLN-1(1-431) plasmids were generated by inserting ephx-1(557-656) and $\operatorname{tn}$-1(1-431) partial cDNAs, respectively, into a pEGFP-C1 vector. The GFP-EPHX-1(557-656; Y568F) plasmid was generated by oligonucleotide-directed inverse PCR using GFP-EPHX-1(557-656) as a template. The pGBD-RHO-1 plasmid was generated by inserting the rho-1 cDNA that lacks the C-terminal CAAX box sequence into the pGBDU vector. $\mathrm{pAD}-\mathrm{EPHX}-1$ and $\mathrm{pAD}-\mathrm{EPHX}-1 \Delta \mathrm{N}$ plasmids were generated by inserting each of the corresponding cDNAs into the pACTII vector. The Pmyo-2::dsred-monomer plasmid was described previously ( $\mathrm{Li}$ et al., 2012).

Generation of mutants using CRISPR-Cas9. pat-3(D768R), ina-1 (R1114D), tln-1(L347A), thn-1(L347A)::CAAX, epac-1(G84E; G440D), and ephx-1(Y568F or Y568E) alleles were generated using the CRISPR (clustered regularly interspaced short palindromic repeats)-Cas9 system, as described previously (Dokshin et al., 2018). CRISPR guide RNAs [5' GUUAUUGAAAGUAGCGUAUU-3' for pat-3(D768R), 5'-UUUCU
UCAAACGAAAUCGUU-3' for ina-1(R1114D), 5' -AGUAACGUUCU UUGUGGUGA-3' for $\operatorname{tn}-1(L 347 A), \quad 5^{\prime}$-CACUUGAAAACAUUAC GAUG-3' for epac-1(G84E), 5'-CUGCGAGAAGGUGAUGAUUU-3' for epac-1(G440D), and 5'-AGCAUAUAAUGUUGAUACAA-3' for $e p h x-1$ (Y568F or Y568E)] and their corresponding $70 \mathrm{nt}$ single-stranded donor template DNAs were synthesized [Integrated DNA Technologies (IDT)], and coinjected with the transactivating CRISPR RNA (IDT), Streptococcus pyogenes Cas9 3NLS (IDT) protein, and pRF4(rol-6d) plasmid into KU501 [for pat-3(D768R), thn-1(L347A), epac-1(G84E; G440D), and $e p h x-1(Y 568 F$ or Y568E)], KU1265 [for pat-3(gk804163; D768R) and pat-3(gk804163) ina-1(R1114D)], and KU1358 [for $t h-1$ (e259); ina-1(R1114D)] strains. The $\operatorname{tn}-1(L 347 A):: C A A X$ allele was created by C-terminal tagging of the $\operatorname{tn}$-1(L347A) allele with the RAP-2 Cterminal region (MNYVQNKSRQSKSCCSLM) containing the CAAX box motif using the CRISPR guide RNA (5'-UUUCUUCAAACGAA AUCGUU-3') and the corresponding $200 \mathrm{nt}$ single-stranded donor template DNA. Each F1 animal carrying the transgene was transferred onto a new dish and single-worm PCR was performed, followed by DNA sequencing to detect mutations. The other strains were generated by standard crosses.

Transgenic animals. Transgenic animals were obtained using the standard C. elegans microinjection method (Mello et al., 1991). Pmyo-2:: dsred-monomer, Punc-25::rap-2(G12V), Punc-25::rap-2(S17A), and Punc-25::eph $x-1 \Delta N$ plasmids were used in kmEx1380 [Punc-25::rap-2 $($ G12V) $(25 \mathrm{ng} / \mu \mathrm{l})+$ Pmyo-2::dsred-monomer $(5 \mathrm{ng} / \mu \mathrm{l})], \quad k m E x 1381$ [Punc-25::rap-2(S17A) $(25 \mathrm{ng} / \mu \mathrm{l})+$ Pmyo-2::dsred-monomer $(5 \mathrm{ng} / \mu \mathrm{l})]$, and kmEx1387 [Punc-25::ephx-1 $\Delta N(25 \mathrm{ng} / \mu \mathrm{l})+$ Pmyo-2::dsred-mono$m e r(5 \mathrm{ng} / \mu \mathrm{l})]$, respectively. The $k m E x 1405$ and $k m E x 1406$ extrachromosomal arrays have been described previously (Shimizu et al., 2018).

Microscopy. Fluorescent images of transgenic animals were observed under the $100 \times$ objective lens of a fluorescence microscope (model ECLIPSE E800, Nikon) and photographed using a CCD camera (Zyla, Oxford Instruments).

Axotomy. Axotomies were performed as described previously (Li et al., 2012). Animals were subjected to axotomy at the young adult stage. Commissures that displayed growth cones or small branches present on the proximal fragment were counted as "regenerated." Proximal fragments that showed no change after $24 \mathrm{~h}$ were counted as "no regeneration." A minimum of 20 individuals with 1-3 one to three axotomized commissures was observed for most experiments.

Biochemical analysis. Transfection of transgenes into COS-7 cells, preparation of the cell lysates, immunoprecipitation, and immunoblotting using anti-FLAG and anti-GFP antibodies has been described previously (Li et al., 2012).

In vitro kinase assays. GFP-EPHX-1(557-656) and GFP-EPHX-1 (557-656; Y568F) proteins were expressed in COS7 cells and immunopurified with an anti-GFP antibody (mouse; stock \#M048-3, MBL). Kinase reactions were performed in a final volume of $20 \mu \mathrm{l}$ buffer consisting of $5 \mathrm{~mm}$ MOPS [3-( $N$-morpholino)propanesulfonic acid], $\mathrm{pH} 7.2$, $2.5 \mathrm{~mm} \beta$-glycerol-phosphate, $5 \mathrm{~mm} \mathrm{MgCl}_{2}, 1 \mathrm{~mm}$ EGTA, $0.5 \mathrm{~mm}$ EDTA, $5 \mu \mathrm{Ci}$ of $\left[\gamma_{-}{ }^{32} \mathrm{P}\right] \mathrm{ATP}, 100 \mu \mathrm{M}$ ATP, and $0.4 \mu \mathrm{g}$ of recombinant $\mathrm{Src}$ (Carna Biosciences). Samples were incubated for $20 \mathrm{~min}$ at $30^{\circ} \mathrm{C}$, and reactions were terminated by the addition of Laemmli sample buffer and boiling. Samples were resolved by SDS-PAGE and analyzed by autoradiography.

Yeast two-hybrid assays. For yeast two-hybrid analysis, GAL4 AD-EPHX-1 or GAL4 AD-EPHX- $1 \Delta \mathrm{N}$ was cotransformed with either GAL4 DBD-RHO-1 or empty pGBDU vectors into the Saccharomyces cerevisiae reporter strain PJ69-4A (MATa trp1-901

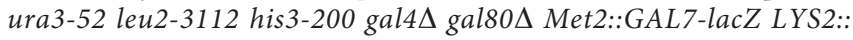
GAL1-HIS3 Ade2::GAL2-ADE2), and yeasts were allowed to grow on SC-Ura-Leu plates. Transformants grown on these plates were then streaked out onto SC-Ura-Leu-His plates with $10 \mathrm{~mm} 5$-aminotriazole and incubated at $30^{\circ} \mathrm{C}$ for $4 \mathrm{~d}$.

Statistical analysis. Statistical analyses were performed as described previously (Li et al., 2012). Briefly, confidence intervals (95\%) were calculated using the modified Wald method and two-tailed $p$ values were calculated using Fisher's exact test (https://www.graphpad.com/ quickcalcs/contingencyl/). 
Table 2. Raw data for genotypes tested by axotomy

\begin{tabular}{|c|c|c|c|c|c|}
\hline Strain & Genotype (juls76 background) & Axons, $n$ & Regenerations, $n$ (\% of total) & $p$ Value & Compared with \\
\hline $\mathrm{KU} 01^{a}$ & Wild type & 74 & $50(68 \%)$ & & \\
\hline $\mathrm{KU} 1265^{a}$ & pat-3(gk804163) & 71 & $23(32 \%)$ & $<0.0001$ & $\mathrm{KU}_{501}{ }^{a}$ \\
\hline KU1356 & 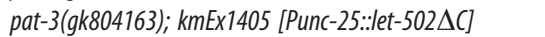 & 79 & $54(68 \%)$ & $<0.0001$ & $\mathrm{KU} 1265^{a}$ \\
\hline KU1357 & pat-3(gk804163); kmEx1406 [Punc-25::venus::m/c-4(DD)] & 62 & $44(71 \%)$ & $<0.0001$ & $\mathrm{KU} 1265^{a}$ \\
\hline $\mathrm{KU}^{\mathrm{N}} 01^{b}$ & Wild type & 55 & $40(73 \%)$ & & \\
\hline $\mathrm{KU} 1358^{a}$ & $\operatorname{tln}-1(e 259)$ & 68 & $23(34 \%)$ & $<0.0001$ & $\mathrm{KU}_{501}{ }^{b}$ \\
\hline $\mathrm{KU} 1265^{b}$ & pat-3(gk804163) & 47 & $17(36 \%)$ & & \\
\hline KU1359 & tln-1(e259); pat-3(gk804163) & 51 & $15(29 \%)$ & $0.6927,0.5226$ & $\mathrm{KU} 1358^{a}, \mathrm{KU} 1265^{b}$ \\
\hline KU1360 & 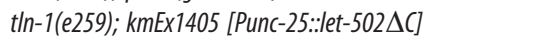 & 67 & $42(63 \%)$ & 0.0010 & $\mathrm{KU} 1358^{a}$ \\
\hline KU1361 & tIn-1(e259); kmEx1406 [Punc-25::venus::m/c-4(DD)] & 49 & $33(67 \%)$ & 0.0004 & $\mathrm{KU} 1358^{a}$ \\
\hline KU501 ${ }^{C}$ & Wild type & 60 & $37(62 \%)$ & & \\
\hline KU1358 ${ }^{b}$ & $\operatorname{tnn}-1(e 259)$ & 46 & $13(28 \%)$ & 0.0008 & KU501' \\
\hline KU1362 & pat-3(D768R) & 44 & $26(59 \%)$ & 0.8406 & KU501' \\
\hline KU1363 & $\operatorname{tln}-1(e 259) ;$ pat-3(D768R) & 62 & $38(61 \%)$ & 0.0009 & KU1358 \\
\hline $\mathrm{KU} 501^{d}$ & Wild type & 37 & $24(65 \%)$ & & \\
\hline KU1364 & ina-1(R1114D) & 60 & $38(63 \%)$ & 1.0000 & $\mathrm{KU}_{501^{d}}$ \\
\hline KU1358 & $\operatorname{tln}-1(e 259)$ & 54 & $17(31 \%)$ & & \\
\hline KU1365 & $\operatorname{tln}-1(e 259) ;$ ina-1(R1114D) & 60 & $36(60 \%)$ & 0.0027 & KU1358 \\
\hline KU1265 & pat-3(gk804163) & 46 & $17(37 \%)$ & & \\
\hline KU1366 & pat-3(gk804163; D768R) & 56 & $36(64 \%)$ & 0.0093 & KU1265 \\
\hline KU1367 & pat-3(gk804163) ina-1(R1114D) & 64 & $41(64 \%)$ & 0.0067 & KU1265 \\
\hline $\mathrm{KU} 501^{e}$ & Wild type & 38 & $24(63 \%)$ & & \\
\hline KU1368 & $\operatorname{rap}-1(p k 2082)$ & 26 & $19(73 \%)$ & 0.4329 & $\mathrm{KU} 501^{e}$ \\
\hline $\mathrm{KU} 1369^{a}$ & $\operatorname{rap}-2(g k 11)$ & 62 & $25(40 \%)$ & 0.0389 & $\mathrm{KU}_{501}{ }^{e}$ \\
\hline $\mathrm{KU} 1358^{d}$ & $\operatorname{tln}-1(e 259)$ & 40 & $12(30 \%)$ & & \\
\hline KU1370 & $\operatorname{tln}-1(e 259) ; \operatorname{rap}-2(g k 11)$ & 49 & $14(29 \%)$ & $0.2327,1.0000$ & $\mathrm{KU}_{1369^{a}}, \mathrm{KU} 1358^{d}$ \\
\hline $\mathrm{KU} 1369^{b}$ & $\operatorname{rap}-2(g k 11)$ & 42 & $14(33 \%)$ & & \\
\hline KU1371 & $\operatorname{tln}-1(L 347 A) ; \operatorname{rap}-2(g k 11)$ & 49 & $19(39 \%)$ & 0.6645 & KU1369 \\
\hline KU1372 & $\operatorname{tln}-1(L 347 A:: C A A X) ; \operatorname{rap}-2(g k 11)$ & 78 & $48(62 \%)$ & 0.0041 & $\mathrm{KU} 1369^{b}$ \\
\hline KU1369' & $\operatorname{rap}-2(g k 11)$ & 29 & $10(34 \%)$ & & \\
\hline KU1373 & pat-3(D768R); rap-2(gk11) & 60 & $21(35 \%)$ & 1.0000 & KU1369 \\
\hline KU1374 & 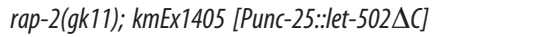 & 55 & $25(45 \%)$ & 0.3618 & KU1369 \\
\hline KU1375 & deb-1(gk329549) & 58 & $21(36 \%)$ & 0.0004 & $\mathrm{KU} 501^{a}$ \\
\hline KU1376 & 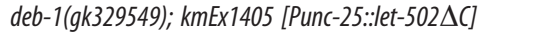 & 66 & $23(35 \%)$ & 1.0000 & KU1375 \\
\hline KU1377 & deb-1(gk329549); kmEx1406 [Punc-25::venus::mlc-4(DD)] & 51 & $21(41 \%)$ & 0.6940 & KU1375 \\
\hline $\mathrm{KU} 01^{f}$ & Wild type & 46 & $30(65 \%)$ & & \\
\hline KU1378 & epac-1(tm3203) & 50 & $19(38 \%)$ & 0.0088 & $\mathrm{KU}_{501}{ }^{f}$ \\
\hline $\mathrm{KU} 1369^{d}$ & rap-2(gk11) & 46 & $16(35 \%)$ & 0.0064 & $\mathrm{KU} 01^{f}$ \\
\hline KU1379 & epac-1(tm3203); rap-2(gk11) & 45 & $19(42 \%)$ & $0.6817,0.5219$ & KU1378, KU1369 \\
\hline KU1380 & epac-1(tm3203); kmEx1380 [Punc-25::rap-2(G12V)] & 59 & $48(81 \%)$ & $<0.0001$ & KU1378 \\
\hline KU1381 & epac-1(tm3203); kmEx1381 [Punc-25::rap-2(S17A)] & 46 & $24(52 \%)$ & 0.2180 & KU1378 \\
\hline $\mathrm{KU} 501^{g}$ & Wild type & 62 & $41(66 \%)$ & & \\
\hline KU1382 & epac-1(G84E; G440D) line \#1 & 59 & $25(42 \%)$ & 0.0108 & $\mathrm{KU}_{50}{ }^{g}$ \\
\hline KU1383 & epac-1(G84E; G440D) line \#2 & 72 & $29(40 \%)$ & 0.0033 & $\mathrm{KU}_{50}{ }^{g}$ \\
\hline $\mathrm{KU} 01^{h}$ & wild type & 41 & $28(68 \%)$ & & \\
\hline KU1384 & ephx-1(Y568F) & 49 & $19(39 \%)$ & 0.0063 & $\mathrm{KU} 501^{h}$ \\
\hline KU1386 & ephx-1(Y568F); kmEx1405 [Punc-25::let-502DC] & 56 & $42(75 \%)$ & 0.0003 & KU1384 \\
\hline KU1385 & ephx-1(Y568E) & 36 & $13(36 \%)$ & 0.0062 & KU501 ${ }^{h}$ \\
\hline $\mathrm{KU}_{501}{ }^{i}$ & Wild type & 50 & $36(72 \%)$ & & \\
\hline KU1387 & 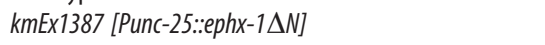 & 26 & $21(81 \%)$ & 0.5776 & $\mathrm{KU} 501^{i}$ \\
\hline KU722 & $\operatorname{src}-1(c j 293)$ & 40 & $9(23 \%)$ & $<0.0001$ & $\mathrm{KU} 51^{i}$ \\
\hline KU1388 & src-1(cj293); kmEx1387 [Punc-25::ephx-1 $\Delta N]$ & 27 & $15(56 \%)$ & 0.0090 & KU722 \\
\hline
\end{tabular}

a to i: different controls of the same strain.

\section{Results}

PAT-3/integrin $\beta$ functions in the RhoA-ROCK-MLC phosphorylation pathway to regulate axon regeneration

C. elegans axon regeneration after axon injury is regulated by the RHO-1/RhoA-LET-502/ROCK-MLC-4 phosphorylation pathway (Fig. 1A; Shimizu et al., 2018). We sought to determine whether integrin functions upstream of the RHO-1 pathway in axon regeneration. We have previously reported that PAT-3/ integrin $\beta$ is involved in axonal regeneration (Hisamoto et al., 2019). Here, we first confirmed the pat-3 mutant phenotype. We subjected GABA-releasing D-type motor neurons (D neurons) to laser axotomy and subsequently monitored the regrowth of their axons. D neurons extend axons from the ventral to dorsal side of the animal body (Fig. 1B). In young adult wild-type animals, $\sim 70 \%$ of severed axons formed growth cones and initiated regeneration within $24 \mathrm{~h}$ after injury (Fig. 1B,C, Table 2). However, in pat-3(gk804163) mutants, the frequency of axon regeneration was substantially reduced (Fig. $1 B, C$, Table 2).

Next, we investigated the relationship between PAT- 3 and the RHO-1 pathway. The activation of the RHO-1 pathway is bypassed by the introduction of constitutively active downstream effectors. The kinase activity of LET-502/ROCK is usually 
autoinhibited by its C-terminal region, and truncation of the C-terminus constitutively activates LET-502 kinase activity (Shimizu et al., 2018). We found that the expression of the LET-502 C-terminal truncated mutant (LET-502 $\Delta \mathrm{C}$ ) from the unc-25 promoter in $\mathrm{D}$ neurons could suppress the regeneration defect of pat-3 (gk804163) mutants (Fig. 1C, Table 2). Overexpression of LET-502 $\Delta \mathrm{C}$ does not promote axon regeneration in wild-type animals (Shimizu et al., 2018). These results suggest that $\mathrm{PAT}-3$ functions upstream of LET-502 in axon regeneration.

RhoA activates myosin II via ROCK, which phosphorylates Thr-18 and Ser-19 of the MLC protein (Amano et al., 1996). Next, we examined whether PAT-3 signaling promotes axon regeneration by inducing the phosphorylation of MLC-4. In C. elegans, MLC-4 is phosphorylated by LET-502 at two highly conserved residues, Thr-17 and Ser-18 (Shimizu et al., 2018). We introduced constitutive phosphomimetic mutations, T17D and $S 18 D$, into the $m l c-4$ gene $[m l c-4(D D)]$ and found that the expression of MLC-4(DD) could suppress the axon regeneration defect observed in pat-3(gk804163) mutants (Fig. 1C, Table 2). Expression of MLC-4(DD) in wild-type animals has no effect on the frequency of axon regeneration (Shimizu et al., 2018). Together, these results suggest that PAT-3 functions as an upstream component in the RHO-1-LET-502-MLC4 phosphorylation pathway to promote axon regeneration.

\section{TLN-1/talin promotes axon}

regeneration by inducing integrin inside-out activation

Integrin function is regulated by insideout signaling, which is controlled by talin (Fig. 2A; Kim et al., 2011). We therefore examined whether $C$. elegans TLN-1/talin is also required for axon regeneration. We found that the frequency of axon regeneration in $\mathrm{D}$ neurons was significantly reduced in $t \ln -1(e 259)$ mutants (Fig. 2B, Table 2). Furthermore, the regeneration defect of $t \ln -1$ (e259); pat-3 ( $g k 804163)$ double mutants was no greater than that of $t \ln -1$ (e259) or pat-3 (gk804163) single mutants (Fig. 2B, Table 2), suggesting that TLN-1 acts in the same functional pathway as PAT-3. Consistent with this, we found that, similar to pat-3(gk804163) mutants, the $t n-1$ defect in axon regeneration was suppressed by the expression of LET-502 $\Delta$ C or MLC-4(DD) from the unc-25 promoter in D-type motor neurons (Fig. $2 B$, Table 2). Thus, it is likely that PAT-3 and TLN-1 function in the RHO-1-LET-502-MLC-4 phosphorylation pathway to control axon regeneration.

A

D
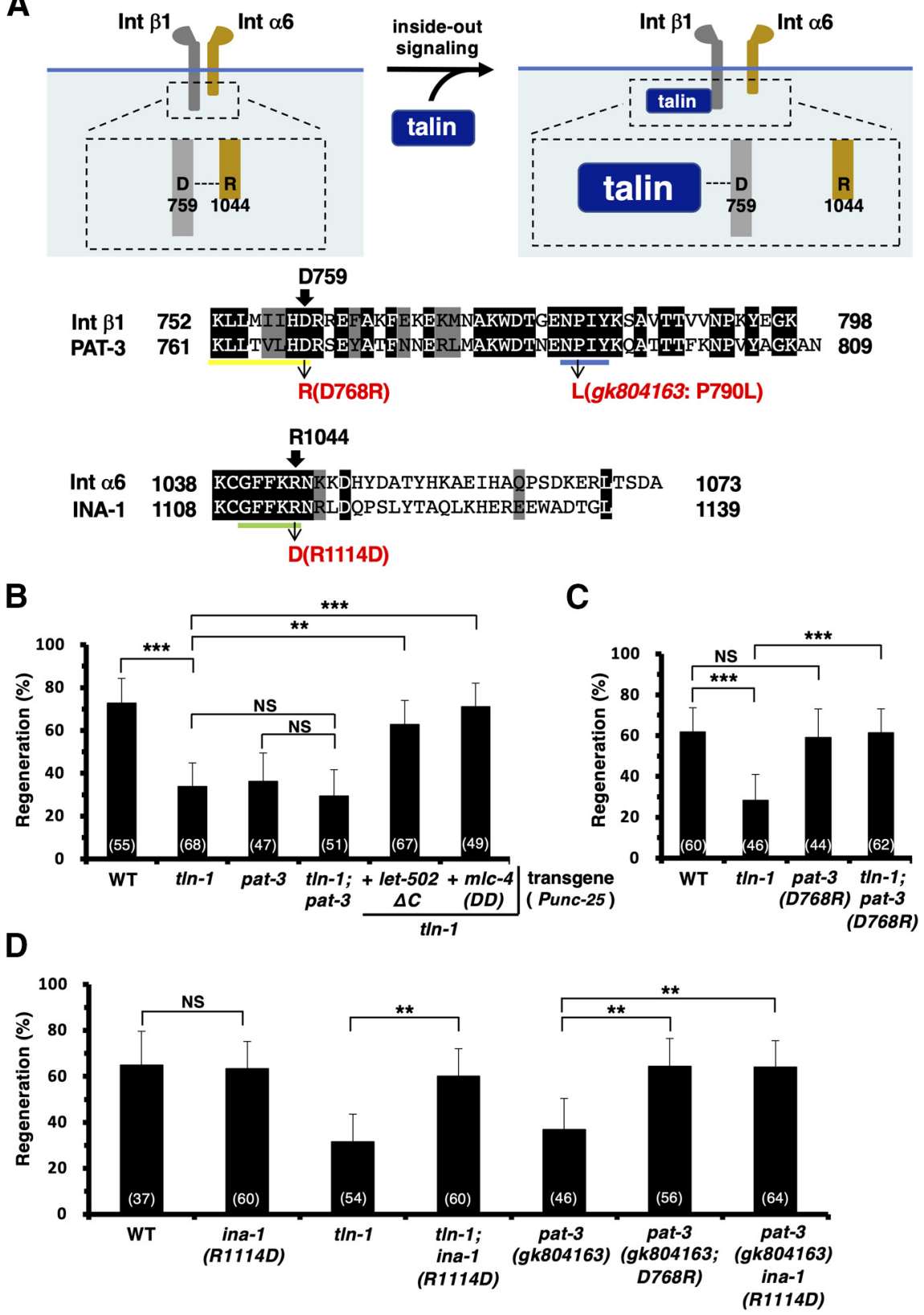

Figure 2. TLN-1 promotes axon regeneration by inducing integrin inside-out activation. $A$, Talin-mediated integrin insideout activation. Talin forms a salt bridge with Asp-759 in $\beta 1$, which disrupts the interaction between $\beta 1$ Asp-759 and $\alpha 6$ Arg-1044. Sequence alignments of cytoplasmic tails between integrin $\beta 1$ and PAT-3 and integrin $\alpha 6$ and INA-1 are shown. Identical and similar residues are highlighted with black and gray shading, respectively. The conserved KLLtVLHD, GFFKR, and NPXY motifs are underlined in yellow, green, and blue, respectively. Asp-759 (in $\beta$ 1), Asp-768 (in PAT-3), Arg-1044 (in $\alpha 6$ ), Arg-1114 (in INA-1), and Pro-790 (in PAT-3) are indicated by arrows. The membranes are shown in blue. $\boldsymbol{B}-\boldsymbol{D}$, Percentages of axons that initiated regeneration $24 \mathrm{~h}$ after laser surgery in the young adult stage. The numbers of axons examined are shown. Error bars indicate $95 \%$ confidence intervals. $* * p<0.01, * * * p<0.001$, as determined by Fisher's exact test. NS, Not significant; WT, wild type; Int, integrin.

In the pat-3(gk804163) mutant, the Pro-790 residue in the membrane-proximal NPxY motif Asn-Pro (790)-Ile-Tyr is replaced with a leucine residue (Fig. 2A; Hisamoto et al., 2019). Integrin activation requires this $\mathrm{NPxY}$ motif in the $\beta$-tail to interact with the PTB (phosphotyrosine-binding) domain in the $\mathrm{N}$-terminal portion of talin (Calderwood et al., 2003). Therefore, the pat-3(gk804163) mutant is expected to be defective in integrin activation by talin binding. Functional integrin receptors are heterodimers composed of one $\alpha$-subunit and one $\beta$-subunit. 
A

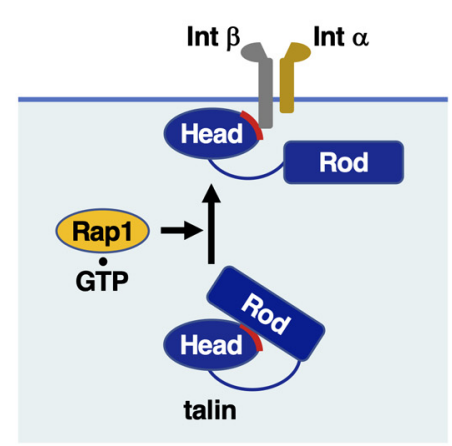

B
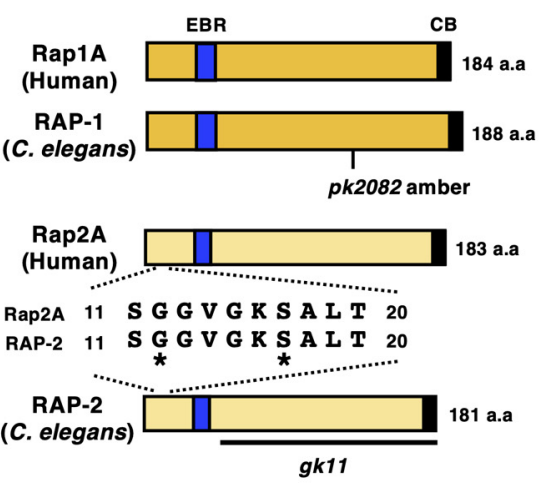

C

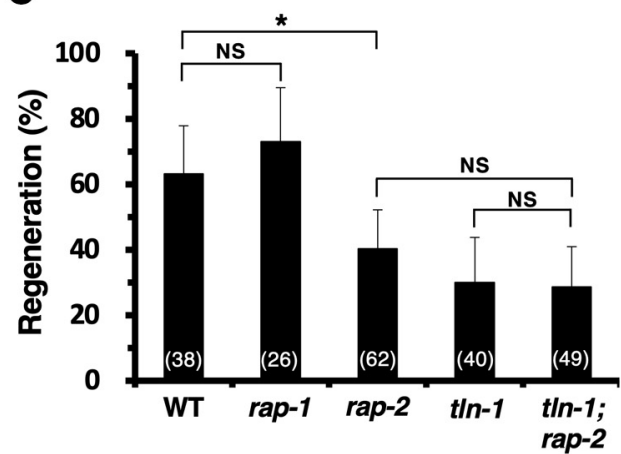

D

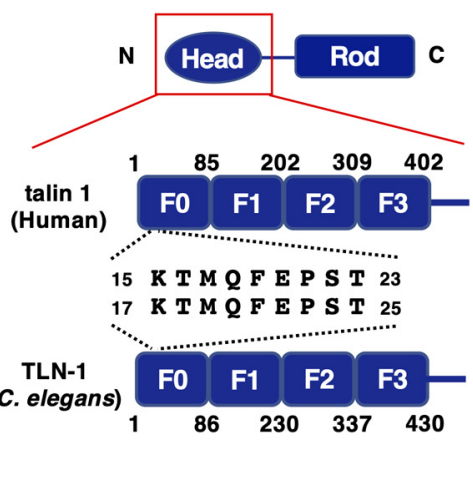

E

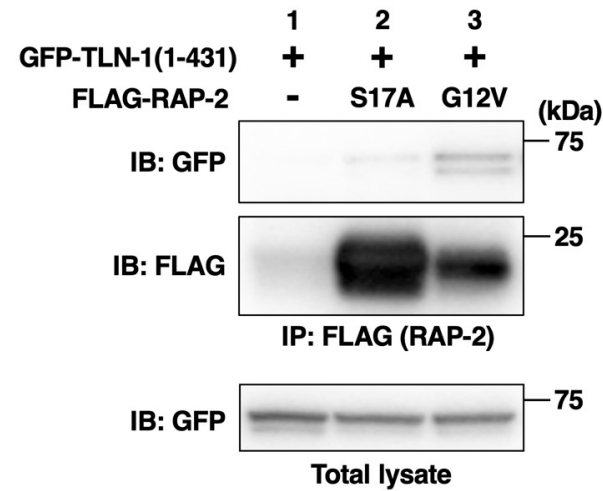

Figure 3. RAP-2 promotes axon regeneration by interacting with TLN-1. A, The Rap1-talin pathway. GTP-bound Rap1 interacts with and recruits talin to the membrane where it is activated, which can expose the integrin $\beta$ tail binding site of talin. B, Structures of RAP-1 and RAP-2. Schematic diagrams of RAP-1, RAP-2, and their human counterparts, Rap1A and Rap2A, are shown. The effector binding region (EBR) is shown in blue, and the CAAX box (CB) is shown in black. Constitutively active RAP-2(G12V) and inactive RAP-2(S17A) mutations are denoted by asterisks. The pk2082 allele of rap- 1 is an amber mutation that generates RAP-1(1-129). Bold line underneath the RAP-2 diagram denotes the extent of the deleted region in the gk11 mutant. C, Percentages of axons that initiated regeneration $24 \mathrm{~h}$ after laser surgery in the young adult stage. The numbers of axons examined are shown. Error bars indicate $95 \%$ confidence intervals. $* p<0.05$, as determined by Fisher's exact test. NS, Not significant. $\boldsymbol{D}$, Structure of the talin head domain. Schematic diagrams of head domains of talin 1 and TLN-1 are shown. The talin head domain is composed of four subdomains: F0, F1, F2, and F3. $E$, Interaction of TLN-1 with RAP-2. COS-7 cells were cotransfected with GFP-TLN-1(1 - 431), and FLAG-GTP-bound RAP-2(G12V) or GDP-bound RAP-2(S17A) as indicated. Complex formation was detected by immunoprecipitation (IP) with anti-FLAG antibody, followed by immunoblotting (IB) with anti-GFP antibody. Total lysates were immunoblotted with anti-GFP antibody. WT, Wild type; Int, integrin.
Therefore, the charge-reversal mutations $D 759 R$ in integrin $\beta 1$ and $R 1044 D$ in integrin $\alpha 6$, wherein Asp-759 and Arg1044 are replaced by arginine and aspartic acid residues, respectively, lead to a constitutively active integrin via the disruption of the $\alpha-\beta$ salt bridge (Fig. 2A; Hughes et al., 1996; Laursen et al., 2011). Because the conserved motifs GFFKR and KLLtVLHD are also present in INA1/integrin $\alpha$ (Arg-1114) and PAT-3/ integrin $\beta$ (Asp-768), respectively (Fig. $2 A)$, we predicted that ina-1(R1114D) and pat-3(D768R) mutations would result in constitutively active integrins. To test this possibility, we generated ina$1(R 1114 D)$ and pat-3(D768R) mutants in the endogenous ina- 1 and pat-3 loci, respectively, by CRISPR/Cas9 mutagenesis. We found that the ina-1(R1114D) and pat-3(D768R) mutations were able to suppress the $t \ln$-1(e259) phenotype of defective axon regeneration (Fig. $2 C$, Table 2 ). These results support the possibility that TLN-1 promotes axon regeneration through integrin inside-out activation.

The $g k 804163$ allele of pat-3 fails to bind to TLN-1 and consequently associates constitutively with INA-1. Thus, the pat-3( $g k 804163)$ mutation is predicted to have a dominant-negative effect on axon regeneration rather than act as a loss-offunction mutation. Consistent with this prediction, we found that the introduction of the $D 768 R$ mutation into the pat3(gk804163) background could suppress the regeneration defect (Fig. 2D, Table 2). Moreover, the ina-1(R1114D) mutation was able to suppress the pat-3 ( $g k 804163$ ) phenotype (Fig. 2D, Table 2). These results suggest that the pat-3 (gk804163) mutation inhibits axon regeneration by constitutively binding to INA-1.

The RAP-2 GTPase-TLN-1 pathway mediates integrin inside-out activation during axon regeneration

What is the mechanism that triggers activation of talin during axon regeneration? Talin is autoinhibited in the cytosol by the interaction of the N-terminal head domain with the $\mathrm{C}$-terminal rod domain, which prevents the interaction of the head domain with the membrane surface and $\beta$-integrin cytoplasmic tail (Goksoy Before integrin activation, they form a salt bridge between Arg (Arg-1044 in integrin $\alpha 6$ ) in the GFFKR motif and Asp (Asp759 in integrin $\beta 1$ ) in the KLLxIIHD motif, which stabilizes the inactive form of integrin (Fig. 2A; Hughes et al., 1996). During integrin inside-out activation, talin binds to the cytoplasmic $\beta$-tail and forms a salt bridge with the conserved Asp residue, which disrupts the inhibitory interaction of the $\beta$-tail with the conserved Arg residue in $\alpha$-integrin (Wegener et al., 2007). et al., 2008). Autoinhibited talin is recruited to the membrane via its freely accessible head domain in a Rap1 GTPase-dependent manner (Zhu et al., 2017). Therefore, Rap1 is essential for integrin inside-out signaling (Fig. 3A). We next examined whether $C$. elegans RAP is also involved in axon regeneration regulated by talin-mediated integrin inside-out activation. The $C$. elegans genome contains the rap-1 and rap-2 genes, which encode the 
A

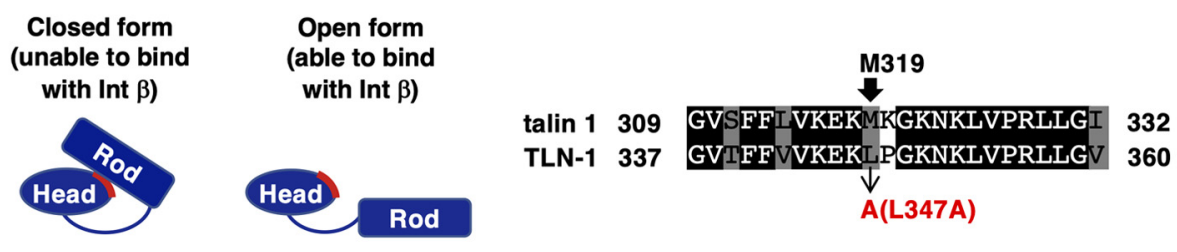

B C

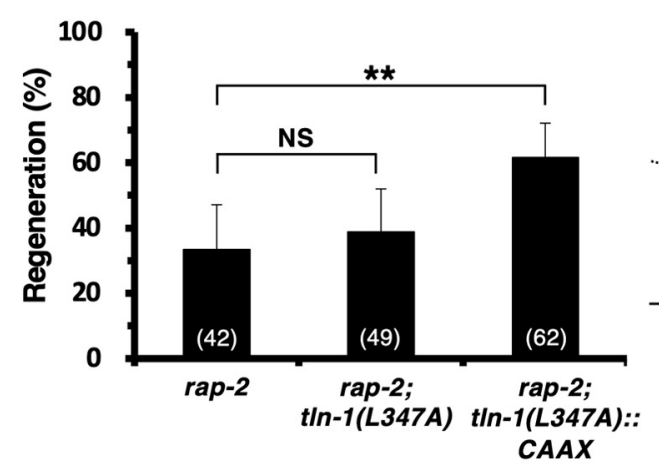

D

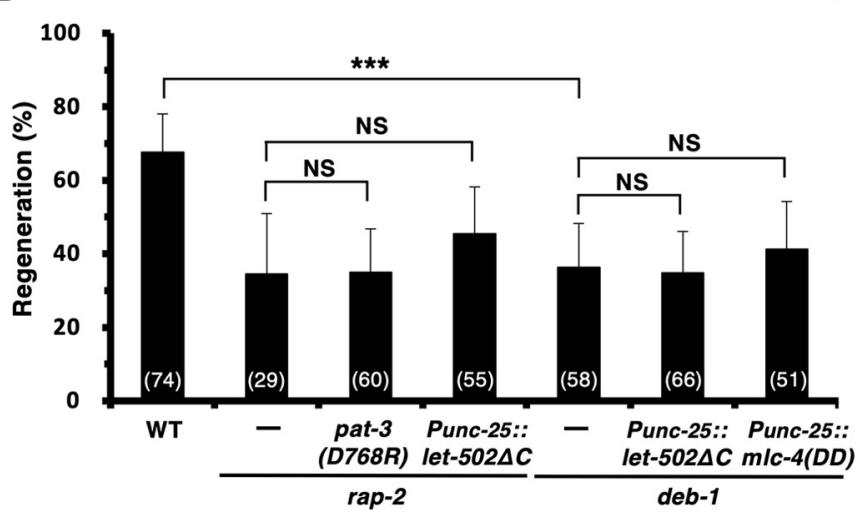

Rap1A 167 RKTPVEKKKPKKKSCLLL 184 RAP-2 164 MNYVQNKSRQSKSCCSLM 181

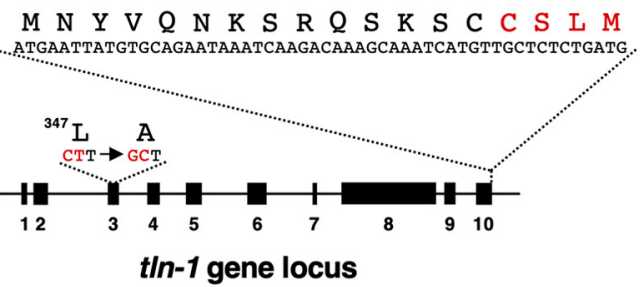

E

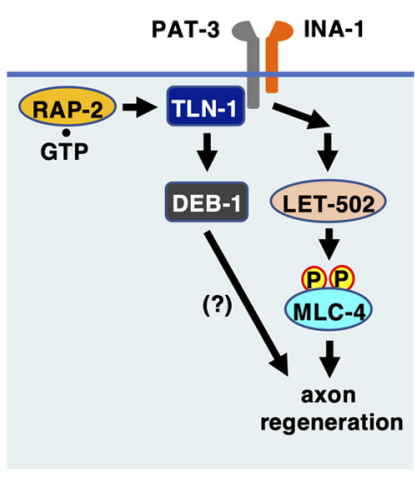

Figure 4. RAP-2 activates TLN-1 in axon regeneration. $A$, Schematic of the domain organization of talin in the open form (right) and the closed, autoinhibited form (left). Talin is autoinhibited by the interaction of the $\mathrm{N}$-terminal head domain with the C-terminal rod domain, which prevents the interaction of the head domain with the membrane surface and $\beta$-integrin cytoplasmic tail. The talin head domain is composed of four subdomains: F0, F1, F2, and F3. Sequence alignment of F3 subdomains between talin 1 and TLN-1 is shown. Identical and similar residues are highlighted with black and gray shading, respectively. The Met-319 site in the talin F3 domain plays a crucial role in interacting with the C-terminal rod domain. The conserved residues Met-319 (in talin 1) and Leu-347 (in TLN-1) are indicated by arrows. B, D, Percentages of axons that initiated regeneration $24 \mathrm{~h}$ after laser surgery in the young adult stage. The numbers of axons examined are shown. Error bars indicate $95 \%$ confidence intervals. $* * p<0.01, * * * p<0.001$, as determined by Fisher's exact test. NS, Not significant. C, Introduction of the RAP-2 CAAX box into the t/n-1 locus. Sequence alignment of CAAX boxes (red line) in Rap1 and RAP-2 is shown. Identical and similar residues are highlighted with black and gray shading, respectively. The C-terminal 18 residues of RAP-2 were inserted by CRISPR/Cas9 mutagenesis into the C-terminal site of the tIn-1 locus carrying the L347A mutation. E, Partners of TLN-1. TLN-1 interacts with PAT-3 and DEB-1. WT, Wild type.

mammalian Rap1 and Rap2 homologs, respectively (Fig. 3B). We found that rap-2(gk11) mutants were defective in axon regeneration, whereas the rap-1(pk2082) mutation had no effect on regeneration (Fig. 3C, Table 2). The regeneration defect observed in rap-2(gk11) mutants was not enhanced by the $\operatorname{tn}$-1(e259) mutation (Fig. 3C, Table 2), suggesting that RAP-2 and TLN-1 act in the same functional pathway.

Several studies have defined a region in talin that binds directly to Rap1 (Zhu et al., 2017; Gingras et al., 2019). The talin head domain is composed of four subdomains, F0, F1, F2, and F3, and the interaction site with Rap1 lies in the F0 domain (Fig. 3D). This region is well conserved between C. elegans TLN-1 and mammalian talin (Fig. 3D). We examined whether RAP-2 binds to the TLN-1 head domain (amino acids 1-431) in a GTP binding-dependent manner. GFP-tagged TLN-1 $(1-431)$ was cotransfected into mammalian COS-7 cells with FLAG-tagged GTP-bound RAP-2(G12V) or GDP-bound
RAP-2(S17A). Coimmunoprecipitation experiments revealed that RAP-2(G12V), but not RAP-2(S17A), interacted with TLN-1 $(1-431)$ (Fig. 3E). These results suggest that direct binding of TLN-1 to RAP-2 via the TLN-1 head domain is evolutionarily conserved and dependent on the GTP-binding form of RAP-2.

Rap1 plays a role in activating talin by recruiting it to the plasma membrane, where the autoinhibitory interaction between the talin $\mathrm{N}$-terminal head and $\mathrm{C}$-terminal rod domains is disrupted, which can expose the integrin $\beta$-tail binding site in talin (Fig. 4A; Gingras et al., 2019). A recent study using nuclear magnetic resonance spectroscopy elucidated the structural basis for talin autoinhibition and revealed that the Met-319 site plays a crucial role in the interaction with the C-terminal rod domain of talin (Goksoy et al., 2008). Therefore, the M319A mutation disrupts the talin autoinhibitory interaction, thereby inducing its constitutively open conformation and activation (Fig. 4A). The 
A
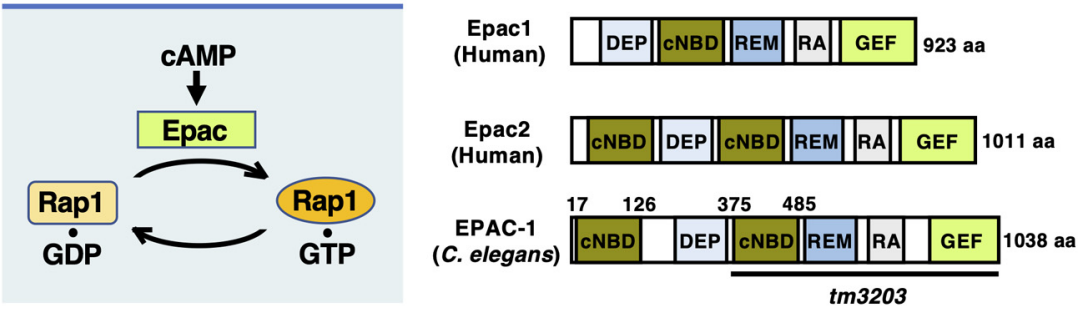

B

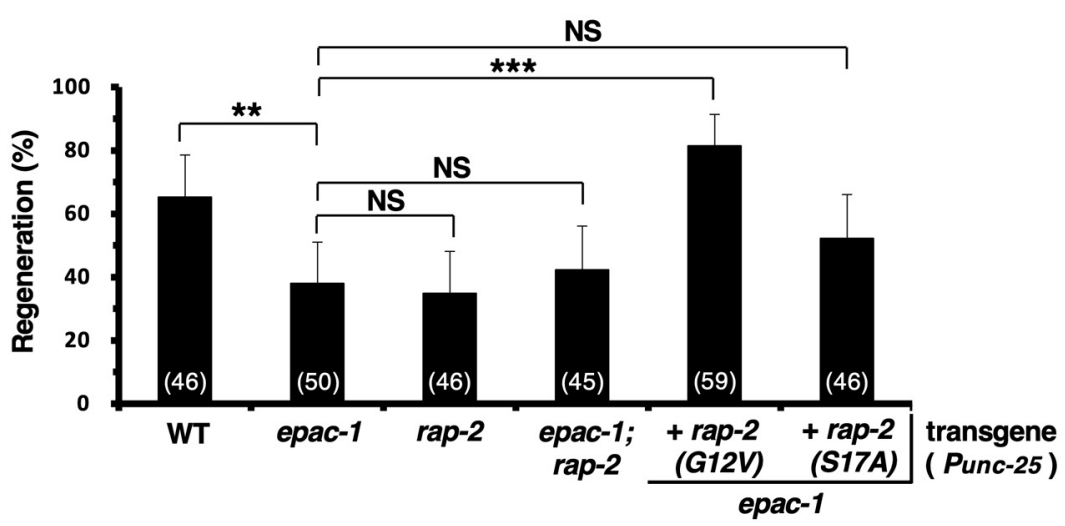

C
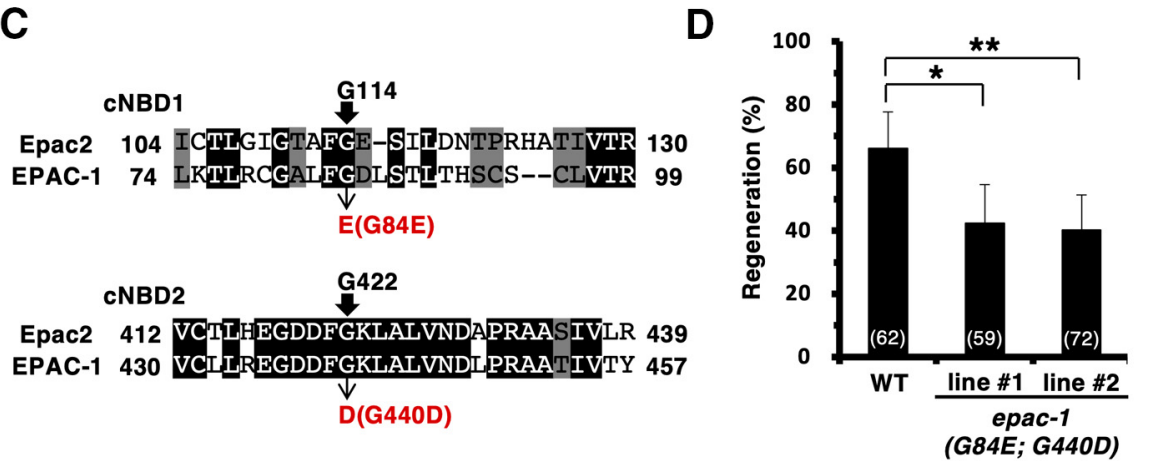

Figure 5. EPAC-1 activates RAP-2 to promote axon regeneration. $\boldsymbol{A}$, Activation of Rap1 by Epac. Schematic diagrams of EPAC-1 and human Epac1 and Epac2 are shown. DEP, Dishevelled, Egl-10, pleckstrin domain; REM, Ras-exchange motif; RA, Ras-associating domain; GEF, GEF catalytic domain. Bold line underneath denotes the extent of the deleted region in the tm3203 mutant. $\boldsymbol{B}, \boldsymbol{D}$, Percentages of axons that initiated regeneration $24 \mathrm{~h}$ after laser surgery in the young adult stage. The numbers of axons examined are shown. Error bars indicate $95 \%$ confidence intervals. $* p<0.05, * * p<0.01$, $* * * p<0.001$, as determined by Fisher's exact test. NS, Not significant. C, CAMP-binding sites in EPAC-1. Sequence alignment of the first and second CNBDs between Epac2 and EPAC-1 is shown. Identical and similar residues are highlighted with black and gray shading, respectively. The Gly-114 and Gly-422 sites in Epac2 are essential for its CAMP binding activity. The conserved residues, Gly-114 (in Epac2), Gly-84 (in EPAC-1), Gly-422 (in Epac2), and Gly-440 (in EPAC-1) are indicated by arrows. WT, Wild type.

region around the Met-319 site in mammalian talin is conserved in C. elegans TLN-1, with the Leu-347 residue corresponding to Met-319 (Fig. 4A). We speculated that the $\ln$-1(L347A) mutation would constitutively activate TLN-1; however, the $t \ln -1$ (L347A) mutation failed to suppress the axon regeneration defect observed in rap-2(gk11) mutants (Fig. 4B, Table 2). Because recruitment of talin to the membrane is required for integrin activation (Goksoy et al., 2008), the ability of the $\ln$-1(L347A) mutation to suppress the rap-2(gk11) defect in axon regeneration may be dependent on membrane localization of TLN-1(L347A). Rapl contains a C-terminal CAAX box that plays an important role in its localization to cellular membranes (Hancock, 2003). To investigate the effect of the membrane localization of TLN-1 (L347A) on the suppression of the rap-2(gk11) phenotype, we used CRISPR/Cas9 mutagenesis to introduce the C-terminal 18 residues of RAP-2, which contain the CAAX box, into the
$\mathrm{COOH}$-terminal site at the $\operatorname{tn}-1$ locus carrying the L347A mutation (Fig. 4C). We found that $\operatorname{tn}-1(L 347 A):: C A A X$ was able to suppress the axon regeneration defect in rap-2(gk11) mutants (Fig. $4 B$, Table 2). These results suggest that TLN-1 (L347A)::CAAX bypasses the requirement for RAP-2 activity to activate integrin in the axon regeneration pathway.

Next, we tested linking RAP-2 to the PAT-3-LET-502/ROCK-MLC-4 phosphorylation pathway in axon regeneration. We found that the regeneration defect in rap-2( $g k 11)$ mutants was not suppressed by the constitutively active pat-3(D768R) mutation or expression of LET-502 $\Delta \mathrm{C}$ in $\mathrm{D}$ neurons (Fig. $4 D$, Table 2). Because RAP-2 regulates axon regeneration by activating TLN-1, TLN1 may mediate signals through two different pathways to promote axon regeneration. A possible second target for a TLN-1-mediated regeneration pathway might be identified by noting that talin also activates vinculin, an essential linker protein between the actin cytoskeleton and ECM-bound integrins (Izard et al., 2004; Parsons et al., 2010). Loveless et al. (2017) demonstrated that the C. elegans vinculin ortholog DEB-1 is associated with TLN-1 (Fig. 4E). Therefore, we examined whether DEB-1 participates in axon regeneration and observed that $d e b-1$ ( $g k 329549)$ mutants were impaired in axon regeneration (Fig. 4D, Table 2). In contrast to pat-3 ( $g k 804163)$ mutants, expression of LET$502 \Delta \mathrm{C}$ or MLC-4(DD) failed to suppress the deb-1(gk329549) phenotype (Fig. 4D, Table 2). These results suggest that TLN-1 regulates axon regeneration via PAT-3- and DEB-1-mediated pathways. Based on these results, it is possible that the $\operatorname{tn}-1$ (e259) mutation is defective in activating the PAT-3 pathway but is capable of activating the DEB-1 pathway.

EPAC-1/Epac activates RAP-2 GTPase to promote axon regeneration

We further evaluated the role of RAP-2 in the axon regeneration pathway. Like other GTPases, Rap exists in inactive GDP-bound and active GTP-bound states. A GEF protein promotes the exchange of GDP for GTP and activates its target GTPase (Boguski and McCormick, 1993). A number of GEFs that mediate the activation of mammalian Rap1 have been identified. An intriguing RapGEF is Epac (exchange protein directly activated by cAMP), because this GEF represents a direct target for cAMP, independent of the classical cAMP target, protein kinase A (PKA; de Rooij et al., 1998; Kawasaki et al., 1998). Indeed, Epac is known to be involved in the control of integrin-mediated cell adhesion (Rangarajan et al., 2003), and cAMP also participates in 
axon regeneration (Bhatt et al., 2004; Pearse et al., 2004; Ghosh-Roy et al., 2010). On the basis of these results, we hypothesized that cAMP-activated Epac is responsible for Rap activation, which leads to talin-mediated integrin activation in axon regeneration (Fig. 5A). The epac-1 gene encodes the $C$. elegans homolog of Epac (Fig. 5A; Tada et al., 2012). We therefore examined whether epac-1 is required for axon regeneration, and we found that epac-1(tm3203) mutants exhibited a phenotype defective in axon regeneration (Fig. 5B, Table 2). We then analyzed the genetic interaction of epac1 with rap-2. We found that epac-1 (tm3203); rap-2(gk11) double mutants were almost as defective in axon regeneration as rap-2(gk11) single mutants (Fig. 5B, Table 2). These results suggest that rap-2 and epac-1 act on the same axis controlling axon regeneration. Furthermore, we found that the expression of constitutively active RAP-2 (G12V), but not inactive RAP-2(S17A) (Fig. $3 B$ ), from the $u n c-25$ promoter in $\mathrm{D}$ type motor neurons could suppress the epac-1 phenotype (Fig. 5B, Table 2), suggesting that EPAC-1 is a GEF for RAP-2 in axon regeneration.

Next, we examined whether cAMP binding is important for EPAC-1 to promote axon regeneration. Epac contains a cyclic nucleotide binding domain (cNBD), which is fused directly to the GEF domain as a single polypeptide chain (Fig. 5A; Bos, 2003). cNBD folds on the GEF domain and prevents its interaction with downstream effectors. Binding of cAMP to Epac results in a conformational change that allows its GEF domain to interact with Rap, leading to Rap activation and subsequent downstream effects. C. elegans EPAC-1 contains two cNBDs, the first at the $\mathrm{N}$ terminus and the second in the middle region (Fig. 5A; Bos, 2003). The human Epac2(G114E; G422D) mutant, wherein both Gly-114 and Gly422 were replaced with glutamic acid and aspartic acid residues, respectively, has been shown to be defective in cAMP binding (Fig. 5C; Ozaki et al., 2000). C. elegans EPAC-1 possesses conserved sites Gly-84 and Gly-440 in the first and second cNBDs, corresponding to Gly-114 and Gly-422 in Epac2, respectively (Fig. 5C). To determine whether the cAMP binding activity of EPAC-1 is important for axon regeneration, we generated epac-1(G84E; G440D) knock-in mutants using the CRISPR/ Cas9 technique. We found that epac-1(G84E; G440D) mutants had significantly reduced axonal regeneration (Fig.

A

\section{C}

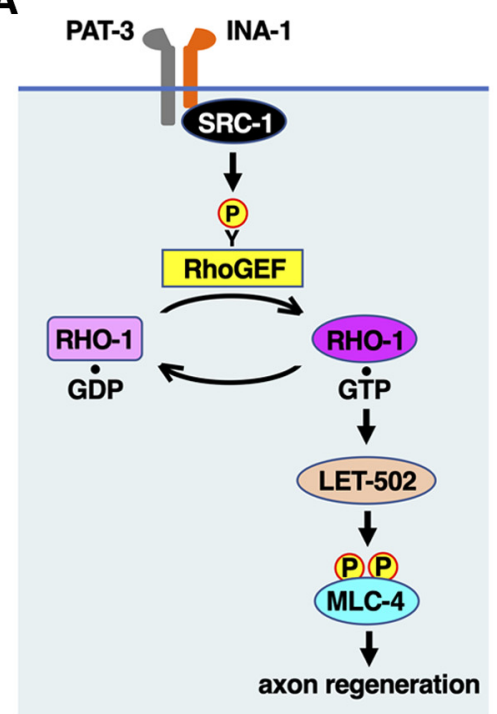

B
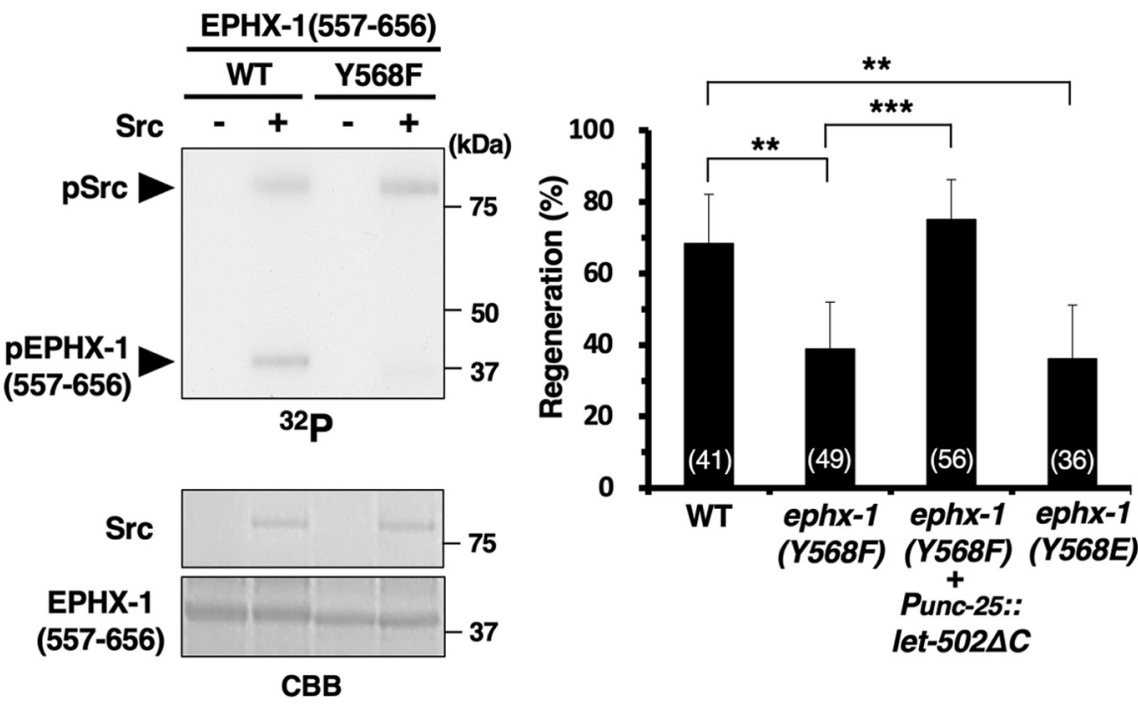

Figure 6. Src phosphorylation of EPHX-1 RhoGEF is essential for axon regeneration. $\boldsymbol{A}$, Activation of the RH0-1-LET-502 pathway by SRC-1 and RhoGEF in axon regeneration. SRC-1 activates RhoGEF by phosphorylating a specific tyrosine residue, which, in turn, leads to the activation of RH0-1. GTP-bound active RH0-1 activates LET-502, resulting in MLC-4 phosphorylation and promoting axon regeneration. $\boldsymbol{B}$, Structure of EPHX-1. Schematic diagrams of EPHX-1 and mammalian ephexin1 are shown. DH domain, yellow; PH domain, blue; SH3 domain, green. Conserved autoinhibitory regions of EPHX-1 and RhoGEFs of the Dbl family are shown in red and highlighted in the multiple-sequence alignment. Identical and similar residues are highlighted with black and gray shading, respectively. Tyr-568 of EPHX-1 is indicated by an asterisk. The EPHX-1(557-656) region is shown (underlined). C, Src phosphorylation of EPHX-1. In vitro phosphorylation of EPHX-1 (557-656) by Src is shown. COS-7 cells were transfected with GFP-EPHX-1(557-656) or GFP-EPHX-1(557-656; Y568F), and cell lysates were immunoprecipitated with an anti-GFP antibody. Immunoprecipitates were incubated with active recombinant $\mathrm{Src}$ in the presence of $\left[\gamma_{-}{ }^{32} \mathrm{P}\right]$ ATP for $20 \mathrm{~min}$ at $30^{\circ} \mathrm{C}$. Autophosphorylated $\mathrm{Src}$ and phosphorylated GFP-EPHX-1(557-656) were resolved by SDS-PAGE (top panel, ${ }^{32} \mathrm{P}$ ). Protein input was confirmed by Coomassie Brilliant Blue (CBB) staining. D, Percentages of axons that initiated regeneration $24 \mathrm{~h}$ after laser surgery in the young adult stage. The numbers of axons examined are shown. Error bars indicate $95 \%$ confidence intervals. $* * p<0.01, * * * p<0.001$, as determined by Fisher's exact test. WT, Wild type.

$5 D$, Table 2), indicating that EPAC-1 is required for axon regeneration in a manner dependent on its cAMP binding activity. Altogether, these results suggest that axon injury leads to increased cAMP levels, which, in turn, activate the EPAC-1-RAP-2-TLN-1-mediated integrin inside-out activation signaling pathway to promote axon regeneration. 
A

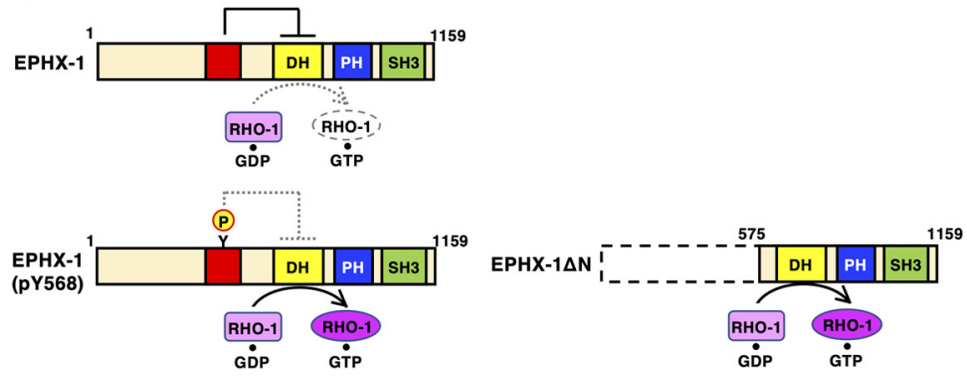

B

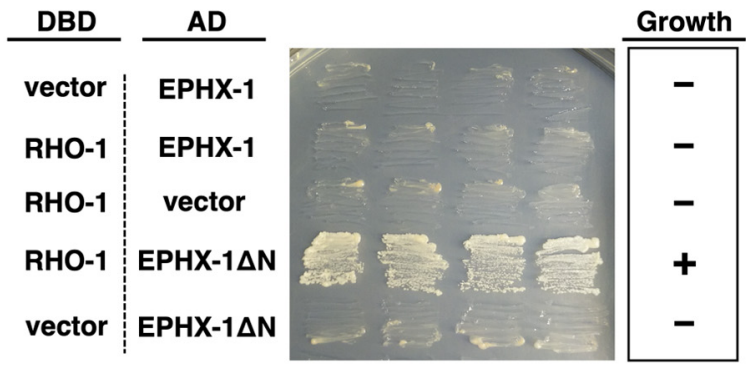

C

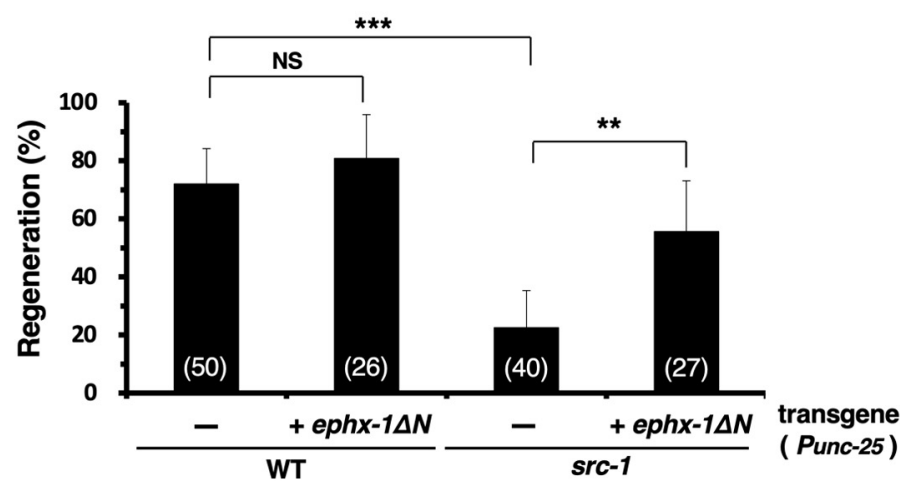

Figure 7. EPHX-1 N-terminal domain inhibits interaction with RH0-1. A, The autoinhibition model for EPHX-1. The autoinhibitory helix (red) packs against a conserved pocket on the DH domain (yellow), which inhibits the interaction between RHO-1 and the DH domain. The tyrosine phosphorylation of the autoinhibitory helix causes its dissociation from the DH domain, resulting in the interaction with RHO-1. EPHX-1 $\Delta \mathrm{N}$ lacking amino acids 1-574 can constitutively associate with RHO1. $B$, Yeast two-hybrid assays for the interactions of RHO-1 with EPHX-1 and EPHX-1 $\triangle N$. The reporter strain PJ69-4A was cotransformed with expression vectors encoding GAL4 DBD-RHO-1, GAL4 AD-EPHX-1, and GAL4 AD-EPHX-1 $\triangle \mathrm{N}$, as indicated. Yeasts carrying the indicated plasmids were grown on selective plates lacking histidine and containing $10 \mathrm{~mm}$-aminotriazole for $4 \mathrm{~d}$. C, Percentages of axons that initiated regeneration $24 \mathrm{~h}$ after laser surgery in the young adult stage. The numbers of axons examined are shown. Error bars indicate $95 \%$ confidence intervals. $* * p<0.01, * * * p<0.001$, as determined by Fisher's exact test. WT, Wild type.

Integrin activates the RhoA signaling pathway via src and RhoGEF in axon regeneration

How does the inside-out activation of integrin regulate the RhoA signaling pathway in axon regeneration? Since RhoA activation depends on RhoGEF activity that catalyzes the GDP-GTP exchange reaction (Cerione and Zheng, 1996), a GEF for the RHO-1 GTPase should function downstream of the integrin signaling pathway in regulating axon regeneration (Fig. 6A). One potential mediator between integrin and RhoA activation is the nonreceptor tyrosine kinase Src. Mammalian Src family kinases can interact directly or indirectly with integrins to activate RhoGEFs of the Dbl family, such as ephexin, through tyrosine phosphorylation (Arias-Salgado et al., 2003; Sahin et al., 2005; Huveneers and Danen, 2009). C. elegans SRC-1/Src interacts directly with INA-1/integrin $\alpha$ and mediates integrin signaling in phagocytic cells (Hsu and $\mathrm{Wu}, 2010$ ). Furthermore, we have previously demonstrated that SRC-1 and INA-1 are required for axon regeneration (Pastuhov et al., 2016). On the basis of these results, we hypothesized that SRC-1 transduces integrin signaling to activate RHO-1 by phosphorylating, thereby activating ephexin-like RhoGEF (Fig. 6A). The C. elegans genome contains EPHX-1, a homolog of mammalian ephexin, that belongs to the Dbl family (Fig. 6B). We therefore investigated the relationship between SRC-1 and EPHX-1 in the axon regeneration pathway. Interestingly, the Src phosphorylation site in ephexin is also conserved in EPHX-1, corresponding to the Tyr-568 residue (Fig. $6 B$ ). To test whether EPHX-1 Tyr-568 is phosphorylated by Src, in vitro kinase assays were performed. Since the full-length recombinant EPHX-1 protein obtained from Escherichia coli was insoluble, a shorter EPHX-1 fragment containing the Tyr-568 site (amino acids 557-656; Fig. 6B) was immunopurified from COS-7 cells expressing GFP-EPHX-1(557-656). We incubated active mammalian Src with EPHX-1(557 - 656) in vitro and observed the phosphorylation of EPHX-1(557656; Fig. 6C). Mutating Tyr-568 to phenylalanine in EPHX-1(557-656) substantially reduced its in vitro phosphorylation by Src (Fig. 6C). These results suggest that EPHX-1 Tyr-568 represents a substrate for direct phosphorylation by Src.

We next addressed the biological importance of EPHX-1 Tyr-568 phosphorylation in axon regeneration. We generated the phosphorylation-defective ephx-1 (Y568F) mutation at the ephx-1 locus by the CRISPR/Cas9 mutagenesis. We found that ephx-1(Y568F) mutants were defective in axon regeneration (Fig. 6D, Table 2). Furthermore, the expression of a constitutively active LET-502 $\Delta \mathrm{C}$ from the unc-25 promoter could suppress the ephx1 (Y568F) mutant phenotype (Fig. 6D, Table 2). To determine whether acidification of the Tyr-568 site would cause constitutive activation, we constructed the phosphomimetic ephx-1 (Y568E) mutation at the ephx-1 locus. However, the ephx-1 (Y568E) mutation lost its ability to promote regeneration (Fig. $6 D$, Table 2), suggesting that the acidic amino acid could not substitute for tyrosine phosphorylation (Hoppmann et al., 2017). Therefore, Tyr-568 phosphorylation of EPHX-1 is necessary for its function as a GEF for RHO-1.

EPHX-1 N-terminal domain inhibits interaction with RHO-1 RhoGEFs of the Dbl family possess tandem Dbl homology (DH), pleckstrin homology $(\mathrm{PH})$, and $\mathrm{SH} 3$ domains (Fig. 7A; Schmidt and Hall, 2002). Many Dbl family proteins are autoinhibited by direct binding of a putative helix $\mathrm{N}$ terminal to the $\mathrm{DH}$ domain, which sterically hinders Rho GTPases and prevents activation (Fig. 7A; Aghazadeh et al., 2000; Yohe et al., 2008). This 
autoinhibition is relieved by the Src phosphorylation of the tyrosine residue in the autoinhibitory helix, which disrupts the interaction with the DH domain. Because Src phosphorylation of EPHX-1 Tyr-568 was required for its function in axon regeneration (Fig. 6D, Table 2), we expected that the deletion of the $\mathrm{N}$ terminus containing Tyr-568 (amino acids 1-574; EPHX-1 $\Delta$ N; Fig. $7 A$ ) would generate a hyperactive EPHX-1. To demonstrate the influence of the EPHX-1 N-terminal domain in regulating its GEF activity, we examined the ability of EPHX- $1 \Delta \mathrm{N}$ to interact with RHO-1 using a yeast two-hybrid system. We found that EPHX-1 $\Delta \mathrm{N}$ formed a complex with RHO-1, whereas full-length EPHX-1 did not associate with RHO-1 (Fig. 7B). These results suggest that the access of $\mathrm{RHO}-1$ to its binding $\mathrm{DH}$ domain is restricted by the $\mathrm{N}$ terminus of EPHX-1.

The increased activity of EPHX- $1 \Delta \mathrm{N}$ was also apparent in testing the epistatic relation between $s r c-1$ and $e p h x-1$ in the regulation of axon regeneration. The expression of $e p h x-1 \Delta N$ from the unc-25 promoter could suppress the regeneration defect of src-1(cj293) mutants (Fig. 7C, Table 2). Based on these findings, we propose that the EPHX-1 N-terminal domain has an autoinhibitory function, which is released by SRC-1 phosphorylation of Tyr-568, resulting in the activation of RHO-1. Thus, SRC-1 functions upstream of EPHX-1 in the RHO-1-LET-502-mediated signaling pathway to regulate axon regeneration.

\section{Discussion}

Integrin has previously been implicated in axonal regeneration in both the peripheral nervous system (PNS) and CNS (Eva and Fawcett, 2014). Successful regeneration in the PNS following injury has been demonstrated correlating with the upregulation of specific integrin subunits, including $\alpha 4, \alpha 5, \alpha 6, \alpha 7$, and $\beta 1$ (Vogelezang et al., 2001; Ekström et al., 2003; Wallquist et al., 2004; Gardiner et al., 2007), suggesting the importance of different types of integrins in neuronal regeneration. In fact, the axonal regeneration of the facial nerve in mice defective in integrin $\alpha 7$ is severely impaired (Werner et al., 2000). In the case of CNS injury, upregulation of inhibitory molecules such as chondroitin sulfate proteoglycans and Nogo-A in a pathologic environment can result in integrin inactivation ( $\mathrm{Hu}$ and Strittmatter, 2008; Tan et al., 2011), whereas induced activation can allow axons to overcome those inhibitory effects on regeneration (Tan et al., 2011). Thus, it is clear that the activation state of integrin is an important factor in achieving significant regenerative growth. However, the molecular mechanisms by which integrin contributes to axonal regeneration have been poorly described. Here, we show that the nonreceptor tyrosine kinase Src and its target ephexin mediate integrin signaling to promote axon regeneration through the RhoA-ROCK-MLC phosphorylation pathway in $C$. elegans. Our findings thus provide a valuable molecular insight into the integrin-regulated repair of damaged neurons.

In this study, we demonstrate that integrin activation is essential for creating a functional transmembrane receptor that can induce downstream cellular effects in axon regeneration. A key step in the inside-out activation of integrin signaling is the binding of talin to the cytoplasmic domain of the $\beta$-subunit (Kim et al., 2011). When the receptor is in the bent, inactivated state, a salt bridge is formed between the KLLxIIHD motif on the $\beta$-subunit and GFFKR motif on the $\alpha$-subunit. When talin binds to the cytoplasmic tail of the $\beta$-subunit, the transmembrane domains of $\alpha$ - and $\beta$-integrins separate, resulting in a conformational change that modulates downstream signaling. The mutated form of the integrin $\beta$-subunit D759R (numbered

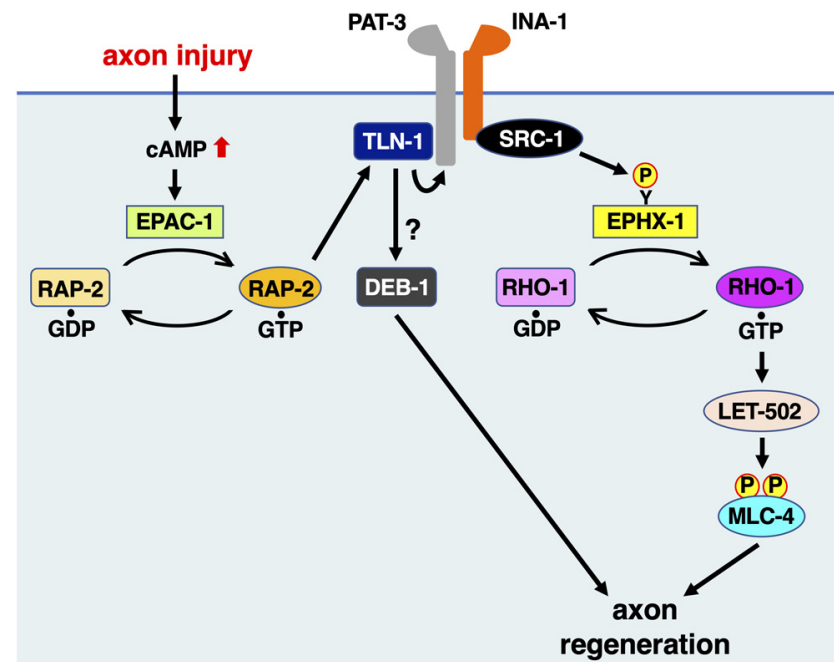

Figure 8. Schematic model for the regulation of axon regeneration by the integrin signaling network. In response to axon injury, CAMP levels are elevated, resulting in the activation of EPAC-1, which, in turn, activates RAP-2. GTP-bound RAP-2 can interact with and activate $T L N-1$, which then activates integrin and possibly DEB-1 as well. Next, integrin activation directs the GDP-GTP exchange activity of EPHX-1 toward RH0-1 via SRC-1-mediated phosphorylation of EPHX-1 on Tyr-568. Finally, GTP-bound RH0-1 activates LET-502, leading to MLC-4 phosphorylation, which promotes axon regeneration.

using the $\beta 1$ sequence) leads to constitutive activation of integrin by disrupting the salt bridge between the $\alpha$ - and $\beta$-subunits (Laursen et al., 2011). The conserved sequences GFFKR and KLLtVLHD are also present in INA-1/integrin $\alpha$ and PAT-3/ integrin $\beta$, respectively. This raises the possibility that talinmediated inside-out activation of integrin may be involved in the regulation of axon regeneration. Indeed, we found that the pat-3, ina-1, and $t \ln -1$ mutants are defective in axon regeneration and that the pat-3(D768R) mutation, corresponding to the mammalian integrin $\beta 1$ (D759R) mutation, can suppress the $\ln -1 \mathrm{mu}-$ tant phenotype, suggesting that the pat-3(D768R) mutation is constitutively active. These results are consistent with the possibility that the TLN-1/talin-mediated activation of PAT-3/integrin $\beta$ promotes axon regeneration (Fig. 8).

How is TLN-1 activated in response to axonal injury? Mammalian talin exists in the cytoplasm in a closed, autoinhibited conformation, in which intramolecular interactions between the N-terminal and C-terminal domains prevent integrin binding (Goksoy et al., 2008). Upon stimulation, talin is efficiently recruited to the cell membrane and released from its autoinhibitory conformation to trigger integrin activation (Song et al., 2012). It has been suggested that the Rap1 GTPase plays a critical role in activating talin by recruiting it to the membrane, relieving the autoinhibitory interactions (Zhu et al., 2017). In addition, Rap1 is activated by GEF Epac1, which is a target of cAMP (de Rooij et al., 1998; Kawasaki et al., 1998). The present study provides compelling evidence that the EPAC-1/Epac-RAP-2 GTPase signaling pathway activates TLN-1/talin-mediated integrin activation in axon regeneration (Fig. 8). In the absence of cAMP, mammalian Epac has been reported to exist in an inactive conformation such that access of Rap1 to the GEF domain of Epac is occluded by an intramolecular interaction between the cAMP-binding domain and catalytic region (Rehmann et al., 2003, 2006). Upon cAMP accumulation, nucleotide binding promotes conformational change in Epac that uncouples the cAMPbinding domain from the catalytic region, enabling Rap1 activation. Thus, the Epac-Rap1 pathway represents a PKA-independent cAMP signaling cascade. The secondary messenger cAMP is 
involved in axonal regeneration through the activation of PKA (Gao et al., 2004). However, an additional cAMP-dependent mechanism involves EPAC-1, which also responds to physiological changes in CAMP concentration, promoting the activation of the RAP-2 GTPase.

Since the artificial localization of TLN-1 to the membrane can suppress the defect in axon regeneration caused by the rap-2 mutation, the main role of RAP-2 in axon regeneration is to recruit TLN-1 to the membrane. However, the constitutively active pat-3(D768R) mutation or LET-502 $\Delta \mathrm{C}$ expression is unable to suppress the rap-2 defect. Thus, the PAT-3-LET-502 pathway does not appear to be simply downstream of RAP-2 signaling, but another pathway is also likely to be important for axon regeneration. A possible factor in this other pathway might be vinculin, an essential linker protein between the actin cytoskeleton and integrins bound to the ECM. Upon activation by talin, vinculin binds to both integrin-bound talin and actin filaments, allowing cells to transmit the force generated by actomyosin to the ECM via the vinculin-talin-integrin complex (Zamir and Geiger, 2001; Izard et al., 2004). In fact, we find that DEB-1/ vinculin is involved in axon regeneration. In contrast to pat-3 mutants, the expression of LET-502 $\Delta \mathrm{C}$ does not suppress the deb-1 mutant defect in axon regeneration. Based on these results, we hypothesize that TLN-1 regulates axon regeneration through PAT-3- and DEB-1-mediated pathways (Fig. 8).

What are mediators of the transduction of integrin signals leading to RHO-1/RhoA GTPase activation? In general, Rho GTPases are activated by RhoGEFs, so we expected that a RhoGEF would be involved in regulating the axon regeneration pathway between integrin and RHO-1. Mammalian integrin $\alpha_{4}-\beta_{1}$ acts via the $\alpha_{4}$ cytoplasmic domain to activate downstream Src, which, in turn, activates the GEF complex that acts on Rac GTPase to promote cell motility (Hsia et al., 2005). These results, combined with our findings, define a conserved integrin signaling pathway, in which activation of SRC-1 by integrin provides a link to RhoGEF-mediated RHO-1 activation during axon regeneration in C. elegans. We demonstrate that RhoGEF EPHX1 acts downstream of SRC- 1 in the integrin-RHO-1 signaling pathway (Fig. 8). EPHX-1 is a homolog of the mammalian RhoGEF ephexin, which belongs to the Dbl family of proteins. The Dbl family GEFs share the structural motif of the central $\mathrm{DH}$ catalytic domain in tandem with the regulatory $\mathrm{PH}$ domain. The N-terminal half of Dbl RhoGEFs has been proven to be characterized by a negative regulatory element for the $\mathrm{DH}-\mathrm{PH}$ functional module (Aghazadeh et al., 2000; Yohe et al., 2007, 2008; Tanegashima et al., 2008). This intramolecular interaction hinders the Rho GTPase access to the DH domain needed to catalyze the guanine nucleotide exchange. Mammalian ephexin is phosphorylated at a tyrosine residue in an $\mathrm{N}$-terminal motif and has considerable sequence identity with the autoinhibitory helix described for the members of the Dbl family (Sahin et al., 2005; Yohe et al., 2008). Upon the phosphorylation of this tyrosine residue in the N-terminal region by Src, Dbl RhoGEFs open to yield an active conformation with an exposed DH domain, relieving this autoinhibition. Indeed, the autoinhibitory helix is conserved between EPHX-1 and Dbl family members, and we observed a similar negative regulation of EPHX-1 by its N-terminal domain, as shown by the hyperactivity of the N-terminal truncation mutant. Src phosphorylation of Tyr-568 in the N-terminal domain of EPHX-1 suggests that this tyrosine phosphorylation induces EPHX-1 activation.

In the mammalian Dbl family neuronal guanine exchange factor (Ngef), Tyr-179 is phosphorylated by Src, and substitution of Tyr-179 with glutamic acid (Y179E) causes constitutive activation of Ngef GEF activity (Yohe et al., 2008). In contrast, the Y568E mutation in EPHX-1 is loss of function, which indicates that tyrosine phosphorylation is important for the GEF activity of EPHX-1. The nucleotide exchange activity of the Dbl family RhoGEFs is autoinhibited by an additional intramolecular interaction between the $\mathrm{N}$ - and C-terminal regions (Yohe et al., 2008). Binding of another protein to the N-terminal region would activate the GEF activity by disrupting this intramolecular interaction. One potential binding partner for EPHX-1 might be SRC-1 itself. Here, after SRC-1 phosphorylates EPHX-1 at Tyr568 , the SH2 domain of SRC-1 associates with pTyr-568 in EPHX-1, resulting in the activation of EPHX-1 GEF activity by relieving the autoinhibitory intramolecular interaction. Because SRC-1 associates with INA-1 (Hsu and Wu, 2010), this possibility also suggests that SRC- 1 acts on EPHX-1 in the vicinity of the integrin receptor. If so, the interaction of TLN-1 with PAT-3 could induce the binding of SRC-1 to INA-1. Therefore, SRC-1 may not only promote GEF activity of EPHX-1, but also determine its subcellular localization.

We have previously shown that the integrin pathway also promotes axon regeneration through activation of the JNK cascade (Pastuhov et al., 2016; Hisamoto et al., 2019). In this pathway, SRC-1 provides a link between INA-1 and the GEF complex CED-2/CrkII, CED-5/DOCK180, and CED-12/ELMO, which activates CED-10/Rac GTPase. GTP-bound CED-10 interacts with and activates the Ste20-related protein kinase MAX-2, which phosphorylates and activates MLK-1 MAPKKK (Pastuhov et al., 2016). In this JNK pathway, integrin is activated by externalized phosphatidylserine generated by axon severing (Hisamoto et al., 2018). Therefore, SRC-1 activates two separate signaling pathways via RhoGEFs, one that leads to activation of the Rac-JNK pathway, and a second that leads to activation of the RhoA-ROCK pathway that is linked to SRC-1 by TLN-1. Moreover, we have recently identified the $C$. elegans tensin protein TNS-1 as an adaptor protein for the SVH-2 Met-like receptor-JNK signaling pathway (Hisamoto et al., 2019). TNS-1 brings PAT-3 in close proximity to SVH-2 by associating with both proteins. Hence, it is likely that TNS1 links SRC-1 to integrin-JNK signaling.

\section{References}

Aghazadeh B, Lowry WE, Huang X-Y, Rosen MK (2000) Structural basis for relief of autoinhibition of the Dbl homology domain of proto-oncogene Vav by tyrosine phosphorylation. Cell 102:625-633.

Amano M, Ito M, Kimura K, Fukata Y, Chihara K, Nakano T, Matsuura Y, Kaibuchi K (1996) Phosphorylation and activation of myosin by Rhoassociated kinase (Rho-kinase). J Biol Chem 271:20246-20249.

Arias-Salgado EG, Lizano S, Sarkar S, Brugge JS, Ginsberg MH, Shattil SJ (2003) Src kinase activation by direct interaction with the integrin cytoplasmic domain. Proc Natl Acad Sci U S A 100:13298-13302.

Bejjani RE, Hammarlund M (2012) Notch signaling inhibits axon regeneration. Neuron 73:268-278.

Bhatt DH, Otto SJ, Depoister B, Fetcho JR (2004) Cyclic AMP-induced repair of Zebrafish spinal circuits. Science 305:254-258.

Bishop AL, Hall A (2000) Rho GTPases and their effector proteins. Biochem J 348:241-255.

Boguski MS, McCormick F (1993) Proteins regulating Ras and its relatives. Nature 366:643-654.

Bos JL (2003) Epac: a new cAMP target and new avenues in cAMP research. Nat Rev Mol Cell Biol 4:733-738.

Bradke F, Fawcett JW, Spira ME (2012) Assembly of a new growth cone after axotomy: the precursor to axon regeneration. Nat Rev Neurosci 13:183193.

Brenner S (1974) The genetics of Caenorhabditis elegans. Genetics 77:71-94 
Calderwood DA, Fujioka Y, de Pereda JM, García-Alvarez B, Nakamoto T, Margolis B, McGlade CJ, Liddington RC, Ginsberg MH (2003) Integrin $\beta$ cytoplasmic domain interactions with phosphotyrosine-binding domains: a structural prototype for diversity in integrin signaling. Proc Natl Acad Sci U S A 100:2272-2277.

Cerione RA, Zheng Y (1996) The Dbl family of oncogenes. Curr Opin Cell Biol 8:216-222.

Coles CH, Bradke F (2015) Coordinating neuronal actin-microtubule dynamics. Curr Biol 25:R677-R691.

Dokshin GA, Ghanta KS, Piscopo KM, Mello CC (2018) Robust genome editing with short single-stranded and long, partially single-stranded DNA donors in Caenorhabditis elegans. Genetics 210:781-787.

Ekström PAR, Mayer U, Panjwani A, Pountney D, Pizzey J, Tonge DA (2003) Involvement of $\alpha 7 \beta 1$ integrin in the conditioning-lesion effect on sensory axon regeneration. Mol Cell Neurosci 22:383-395.

Eva R, Fawcett J (2014) Integrin signalling and traffic during axon growth and regeneration. Curr Opin Neurobiol 27:179-185.

Gao Y, Deng K, Hou J, Bryson JB, Barco A, Nikulina E, Spencer T, Mellado W, Kandel ER, Filbin MT (2004) Activated CREB Is sufficient to overcome inhibitors in myelin and promote spinal axon regeneration In vivo. Neuron 44:609-621.

Gardiner NJ, Moffatt S, Fernyhough P, Humphries MJ, Streuli CH, Tomlinson DR (2007) Preconditioning injury-induced neurite outgrowth of adult rat sensory neurons on fibronectin is mediated by mobilisation of axonal $\alpha 5$ integrin. Mol Cell Neurosci 35:249-260.

Ghosh-Roy A, Wu Z, Goncharov A, Jin Y, Chisholm AD (2010) Calcium and cyclic AMP promote axonal regeneration in Caenorhabditis elegans and require DLK-1 kinase. J Neurosci 30:3175-3183.

Gingras AR, Lagarrigue F, Cuevas MN, Valadez AJ, Zorovich M, McLaughlin W, Lopez-Ramirez MA, Seban N, Ley K, Kiosses WB, Ginsberg MH (2019) Rap1 binding and a lipid-dependent helix in talin F1 domain promote integrin activation in tandem. J Cell Biol 218:17991809.

Goksoy E, Ma Y-Q, Wang X, Kong X, Perera D, Plow EF, Qin J (2008) Structural basis for the autoinhibition of talin in regulating integrin activation. Mol Cell 31:124-133.

Hall A (1998) Rho GTPases and the actin cytoskeleton. Science 279:509-514.

Hammarlund M, Nix P, Hauth L, Jorgensen EM, Bastiani M (2009) Axon regeneration requires a conserved MAP kinase pathway. Science 323:802-806.

Hancock JF (2003) Ras proteins: different signals from different locations. Nat Rev Mol Cell Biol 4:373-384.

He Z, Jin Y (2016) Intrinsic control of axon regeneration. Neuron 90:437451.

Hisamoto N, Tsuge A, Pastuhov SI, Shimizu T, Hanafusa H, Matsumoto K (2018) Phosphatidylserine exposure mediated by ABC transporter activates the integrin signaling pathway promoting axon regeneration. Nat Commun 9:3099.

Hisamoto N, Shimizu T, Asai K, Sakai Y, Pastuhov SI, Hanafusa H, Matsumoto K (2019) C. elegans tensin promotes axon regeneration by linking the Met-like SVH-2 and integrin signaling pathways. J Neurosci 39:5662-5672.

Hoppmann C, Wong A, Yang B, Li S, Hunter T, Shokat KM, Wang L (2017) Site-specific incorporation of phosphotyrosine using an expanded genetic code. Nat Chem Biol 13:842-844.

Hsia DA, Lim S-T, Bernard-Trifilo JA, Mitra SK, Tanaka S, den Hertog J, Streblow DN, Ilic D, Ginsberg MH, Schlaepfer DD (2005) Integrin $\alpha 4 \beta 1$ promotes focal adhesion kinase-independent cell motility via $\alpha 4$ cytoplasmic domain-specific activation of c-Src. Mol Cell Biol 25:9700-9712.

Hsu T-Y, Wu Y-C (2010) Engulfment of apoptotic cells in C. elegans Is mediated by integrin $\alpha$ /SRC Signaling. Curr Biol 20:477-486.

Hu F, Strittmatter SM (2008) The N-terminal domain of Nogo-A inhibits cell adhesion and axonal outgrowth by an integrin-specific mechanism. J Neurosci 28:1262-1269.

Hughes PE, Diaz-Gonzalez F, Leong L, Wu C, McDonald JA, Shattil SJ, Ginsberg MH (1996) Breaking the integrin hinge. A defined structural constraint regulates integrin signaling. J Biol Chem 271:6571-6574.

Huveneers S, Danen EHJ (2009) Adhesion signaling - crosstalk between integrins, Src and Rho. J Cell Sci 122:1059-1069.

Hynes RO (1987) Integrins: a family of cell surface receptors. Cell 48:549554.
Izard T, Evans G, Borgon RA, Rush CL, Bricogne G, Bois PRJ (2004) Vinculin activation by talin through helical bundle conversion. Nature 427:171-175.

Kawasaki H, Springett GM, Mochizuki N, Toki S, Nakaya M, Matsuda M, Housman DE, Graybiel AM (1998) A family of cAMP-binding proteins that directly activate Rap1. Science 282:2275-2279.

Kim C, Ye F, Ginsberg MH (2011) Regulation of integrin activation. Annu Rev Cell Dev Biol 27:321-345.

Kim M, Carman CV, Springer TA (2003) Bidirectional transmembrane signaling by cytoplasmic domain separation in integrins. Science 301:17201725.

Laursen LS, Chan CW, ffrench-Constant C (2011) Translation of myelin basic protein mRNA in oligodendrocytes is regulated by integrin activation and hnRNP-K. J Cell Biol 192:797-811.

Li C, Hisamoto N, Nix P, Kanao S, Mizuno T, Bastiani M, Matsumoto K (2012) The growth factor SVH-1 regulates axon regeneration in C. elegans via the JNK MAPK cascade. Nat Neurosci 15:551-557.

Loveless T, Qadota H, Benian GM, Hardin J (2017) Caenorhabditis elegans SORB-1 localizes to integrin adhesion sites and is required for organization of sarcomeres and mitochondria in myocytes. Mol Biol Cell 28:3621-3633

Mello CC, Kramer JM, Stinchcomb D, Ambros V (1991) Efficient gene transfer in C. elegans: extrachromosomal maintenance and integration of transforming sequences. EMBO J 10:3959-3970.

Nikonenko I, Toni N, Moosmayer M, Shigeri Y, Muller D, Sargent Jones L (2003) Integrins are involved in synaptogenesis, cell spreading, and adhesion in the postnatal brain. Brain Res Dev Brain Res 140:185-194.

Nix P, Hisamoto N, Matsumoto K, Bastiani M (2011) Axon regeneration requires coordinate activation of p38 and JNK MAPK pathways. Proc Natl Acad Sci U S A 108:10738-10743.

Ozaki N, Shibasaki T, Kashima Y, Miki T, Takahashi K, Ueno H, Sunaga Y, Yano H, Matsuura Y, Iwanaga T, Takai T, Seino S (2000) cAMP-GEFII is a direct target of cAMP in regulated exocytosis. Nat Cell Biol 2:805-811.

Parsons JT, Horwitz AR, Schwartz MA (2010) Cell adhesion: integrating cytoskeletal dynamics and cellular tension. Nat Rev Mol Cell Biol 11:633643.

Pastuhov SIv, Fujiki K, Tsuge A, Asai K, Ishikawa S, Hirose K, Matsumoto K, Hisamoto N (2016) The core molecular machinery used for engulfment of apoptotic cells regulates the JNK pathway mediating axon regeneration in Caenorhabditis elegans. J Neurosci 36:9710-9721.

Pearse DD, Pereira FC, Marcillo AE, Bates ML, Berrocal YA, Filbin MT, Bunge MB (2004) cAMP and Schwann cells promote axonal growth and functional recovery after spinal cord injury. Nat Med 10:610-616.

Rangarajan S, Enserink JM, Kuiperij HB, de Rooij J, Price LS, Schwede F, Bos JL (2003) Cyclic AMP induces integrin-mediated cell adhesion through Epac and Rap1 upon stimulation of the $\beta 2$-adrenergic receptor. J Cell Biol 160:487-493.

Rehmann H, Prakash B, Wolf E, Rueppel A, de Rooij J, Bos JL, Wittinghofer A (2003) Structure and regulation of the cAMP-binding domains of Epac2. Nat Struct Biol 10:26-32.

Rehmann H, Das J, Knipscheer P, Wittinghofer A, Bos JL (2006) Structure of the cyclic-AMP-responsive exchange factor Epac2 in its auto-inhibited state. Nature 439:625-628.

de Rooij J, Zwartkruis FJT, Verheijen MHG, Cool RH, Nijman SMB, Wittinghofer A, Bos JL (1998) Epac is a Rap1 guanine-nucleotideexchange factor directly activated by cyclic AMP. Nature 396:474-477.

Sahin M, Greer PL, Lin MZ, Poucher H, Eberhart J, Schmidt S, Wright TM, Shamah SM, O'connell S, Cowan CW, Hu L, Goldberg JL, Debant A, Corfas G, Krull CE, Greenberg ME (2005) Eph-dependent tyrosine phosphorylation of ephexin1 modulates growth cone collapse. Neuron 46:191-204.

Schmidt A, Hall A (2002) Guanine nucleotide exchange factors for Rho GTPases: turning on the switch. Genes Dev 16:1587-1609.

Shimizu T, Pastuhov SIv, Hanafusa H, Matsumoto K, Hisamoto N (2018) The C. elegans BRCA2-ALP/Enigma complex regulates axon regeneration via a Rho GTPase-ROCK-MLC phosphorylation pathway. Cell Rep 24:1880-1889.

Song X, Yang J, Hirbawi J, Ye S, Perera HD, Goksoy E, Dwivedi P, Plow EF, Zhang R, Qin J (2012) A novel membrane-dependent on/off switch mechanism of talin FERM domain at sites of cell adhesion. Cell Res 22:1533-1545. 
Tada M, Gengyo-Ando K, Kobayashi T, Fukuyama M, Mitani S, Kontani K, Katada T (2012) Neuronally expressed Ras-family GTPase Di-Ras modulates synaptic activity in Caenorhabditis elegans. Genes Cells 17:778-789.

Tan CL, Kwok JCF, Patani R, ffrench-Constant C, Chandran S, Fawcett JW (2011) Integrin activation promotes axon growth on inhibitory chondroitin sulfate proteoglycans by enhancing integrin signaling. J Neurosci 31:6289-6295.

Tanegashima K, Zhao H, Dawid IB (2008) WGEF activates Rho in the WntPCP pathway and controls convergent extension in Xenopus gastrulation. EMBO J 27:606-617.

Vogelezang MG, Liu Z, Relvas JB, Raivich G, Scherer SS, ffrench-Constant C (2001) $\alpha 4$ integrin is expressed during peripheral nerve regeneration and enhances neurite outgrowth. J Neurosci 21:6732-6744.

Wallquist W, Zelano J, Plantman S, Kaufman SJ, Cullheim S, Hammarberg $H$ (2004) Dorsal root ganglion neurons up-regulate the expression of laminin-associated integrins after peripheral but not central axotomy. J Comp Neurol 480:162-169.

Wegener KL, Partridge AW, Han J, Pickford AR, Liddington RC, Ginsberg MH, Campbell ID (2007) Structural basis of integrin activation by talin. Cell 128:171-182.
Werner A, Willem M, Jones LL, Kreutzberg GW, Mayer U, Raivich G (2000) Impaired axonal regeneration in $\alpha 7$ integrin-deficient mice. J Neurosci 20:1822-1830

Yanik MF, Cinar H, Cinar HN, Chisholm AD, Jin Y, Ben-Yakar A (2004) Functional regeneration after laser axotomy. Nature 432:822-822

Yohe ME, Rossman KL, Gardner OS, Karnoub AE, Snyder JT, Gershburg S, Graves LM, Der CJ, Sondek J (2007) Auto-inhibition of the Dbl family protein tim by an N-terminal helical motif. J Biol Chem 282:1381313823.

Yohe ME, Rossman K, Sondek J (2008) Role of the C-terminal SH3 domain and N-terminal tyrosine phosphorylation in regulation of Tim and related Dbl-family proteins. Biochemistry 47:6827-6839.

Zamir E, Geiger B (2001) Molecular complexity and dynamics of cell-matrix adhesions. J Cell Sci 114:3583-3590.

Zhu L, Yang J, Bromberger T, Holly A, Lu F, Liu H, Sun K, Klapproth S, Hirbawi J, Byzova TV, Plow EF, Moser M, Qin J (2017) Structure of Rap $1 b$ bound to talin reveals a pathway for triggering integrin activation. Nat Commun 8:1744. 